\title{
L-R-smash products and L-R-twisted tensor products of algebras *
}

\author{
Mădălin Ciungu \\ University of Bucharest, Faculty of Mathematics and Informatics \\ Str. Academiei 14, RO-010014 Bucharest 1, Romania \\ Florin Panaite \\ Institute of Mathematics of the Romanian Academy \\ PO-Box 1-764, RO-014700 Bucharest, Romania \\ e-mail: Florin.Panaite@imar.ro
}

\begin{abstract}
We introduce a common generalization of the L-R-smash product and twisted tensor product of algebras, under the name L-R-twisted tensor product of algebras. We investigate some properties of this new construction, for instance we prove a result of the type "invariance under twisting" and we show that under certain circumstances L-R-twisted tensor products of algebras may be iterated.
\end{abstract}

\section{Introduction}

The L-R-smash product over a cocommutative Hopf algebra was introduced and studied in a series of papers [1, [2], 3], 44, with motivation and examples coming from the theory of deformation quantization. This construction was generalized in [15] to the case of arbitrary bialgebras (even quasi-bialgebras), as follows: if $H$ is a bialgebra, $\mathcal{A}$ an $H$-bimodule algebra and $\mathbb{A}$ an $H$-bicomodule algebra, the L-R-smash product $\mathcal{A} \curvearrowleft \mathbb{A}$ is an associative algebra structure defined on $\mathcal{A} \otimes \mathbb{A}$ by the multiplication rule $(\varphi \curvearrowleft u)\left(\varphi^{\prime} \natural u^{\prime}\right)=\left(\varphi \cdot u_{<1>}^{\prime}\right)\left(u_{[-1]} \cdot \varphi^{\prime}\right) \natural u_{[0]} u_{<0>}^{\prime}$, for all $\varphi, \varphi^{\prime} \in \mathcal{A}$ and $u, u^{\prime} \in \mathbb{A}$. The usual smash product $A \# H$ (where $A$ is a left $H$-module algebra) is a particular case, namely $A \# H=A \natural H$ if we regard $A$ as an $H$-bimodule algebra with trivial right $H$-action (and $H$ with its canonical $H$-bicomodule algebra structure).

On the other hand, if $A, B$ are associative algebras and $R: B \otimes A \rightarrow A \otimes B$ is a linear map satisfying certain axioms, then $A \otimes B$ becomes an associative algebra with a multiplication defined in terms of $R$ and the multiplications of $A$ and $B$. This construction appeared in a number of contexts and under different names. Following [8], we call such an $R$ a twisting map and the algebra structure on $A \otimes B$ afforded by it the twisted tensor product of $A$ and $B$ and denote it by $A \otimes_{R} B$. The twisted tensor product of algebras may be regarded as a representative for the cartesian product of noncommutative spaces, better suited than the ordinary tensor product, see [8], 11], [13] for a detailed discussion and references. There are many examples of twisted tensor products of algebras (see for instance [9, [12 for some concrete examples and classification results), the most relevant for us here being the usual smash product $A \# H$ (where $H$ is a bialgebra and $A$ is a left $H$-module algebra).

\footnotetext{
* Research partially supported by the CNCSIS project "Hopf algebras, cyclic homology and monoidal categories", contract nr. 560/2009, CNCSIS code ID_69.
} 
If we look at a general L-R-smash product $\mathcal{A} \curvearrowleft \mathbb{A}$, we realize quickly that it is not an example of a twisted tensor product of algebras. It appears thus natural to try to find a more general construction able to include the L-R-smash product as a particular case; that is, to find a common generalization for the L-R-smash product and the twisted tensor product of algebras. We introduce such a construction in this paper, under the name $L$-R-twisted tensor product of algebras, denoted by $A_{Q} \otimes_{R} B$, where $A, B$ are algebras and $R: B \otimes A \rightarrow A \otimes B, Q: A \otimes B \rightarrow$ $A \otimes B$ are linear maps satisfying certain compatibility conditions. If $Q=i d_{A \otimes B}$, then $A_{Q} \otimes_{R} B$ coincides with the twisted tensor product $A \otimes_{R} B$.

We find then a number of properties of this new construction, inspired by properties of the L-R-smash product or by properties of the twisted tensor product of algebras (or both). For instance, we prove that an L-R-twisted tensor product $A_{Q} \otimes_{R} B$ with bijective $Q$ is isomorphic to a certain twisted tensor product $A \otimes_{P} B$, generalizing a result from [15] stating that an L-R-smash product over a Hopf algebra with bijective antipode is isomorphic to a so-called diagonal crossed product. Also, by generalizing the corresponding result for twisted tensor products proved in [11, we show that under certain circumstances L-R-twisted tensor products may be iterated (but for achieving this, we prove first a result of this type for L-R-smash products that will serve as a guiding example). Finally, we prove a result of the type "invariance under twisting" for L-R-twisted tensor products of algebras, as a common generalization of the corresponding one for twisted tensor products proved in [1] and of an invariance under twisting for L-R-smash products that we prove here and use also as a guiding example.

\section{Preliminaries}

In this section we recall some definitions and results and fix notation that will be used throughout the paper. We work over a commutative field $k$. All algebras, linear spaces etc. will be over $k$; unadorned $\otimes$ means $\otimes_{k}$. By "algebra" we always mean an associative unital algebra.

We recall from [8], 17] that, given two algebras $A, B$ and a $k$-linear map $R: B \otimes A \rightarrow A \otimes B$, with notation $R(b \otimes a)=a_{R} \otimes b_{R}$, for $a \in A, b \in B$, satisfying the conditions

$$
\begin{aligned}
& a_{R} \otimes 1_{R}=a \otimes 1, \quad 1_{R} \otimes b_{R}=1 \otimes b, \\
& \left(a a^{\prime}\right)_{R} \otimes b_{R}=a_{R} a_{r}^{\prime} \otimes b_{R_{r}}, \\
& a_{R} \otimes\left(b b^{\prime}\right)_{R}=a_{R_{r}} \otimes b_{r} b_{R}^{\prime},
\end{aligned}
$$

for all $a, a^{\prime} \in A$ and $b, b^{\prime} \in B$ (where $r$ is another copy of $R$ ), if we define on $A \otimes B$ a new multiplication, by $(a \otimes b)\left(a^{\prime} \otimes b^{\prime}\right)=a a_{R}^{\prime} \otimes b_{R} b^{\prime}$, then this new multiplication is associative and has unit $1 \otimes 1$. In this case, the map $R$ is called a twisting map between $A$ and $B$ and the new algebra structure on $A \otimes B$ is denoted by $A \otimes_{R} B$ and called the twisted tensor product of $A$ and $B$ afforded by $R$.

Let $H$ be a bialgebra, $\mathcal{A}$ an $H$-bimodule algebra (with $H$-module structures denoted by $h \otimes \varphi \mapsto h \cdot \varphi$ and $\varphi \otimes h \mapsto \varphi \cdot h$ for all $h \in H, \varphi \in \mathcal{A}$ ) and $\mathbb{A}$ an $H$-bicomodule algebra (with $H$ comodule structures denoted by $\mathbb{A} \rightarrow H \otimes \mathbb{A}, u \mapsto u_{[-1]} \otimes u_{[0]}$ and $\mathbb{A} \rightarrow \mathbb{A} \otimes H, u \mapsto u_{<0>} \otimes u_{<1>}$ for all $u \in \mathbb{A})$. Define on $\mathcal{A} \otimes \mathbb{A}$ the product $(\varphi \curvearrowleft u)\left(\varphi^{\prime} \natural u^{\prime}\right)=\left(\varphi \cdot u_{<1>}^{\prime}\right)\left(u_{[-1]} \cdot \varphi^{\prime}\right) \natural u_{[0]} u_{<0>}^{\prime}$, for all $\varphi, \varphi^{\prime} \in \mathcal{A}$ and $u, u^{\prime} \in \mathbb{A}$. Then, by [15], this product defines on $\mathcal{A} \otimes \mathbb{A}$ a structure of associative algebra with unit $1_{\mathcal{A}} \otimes 1_{\mathbb{A}}$, denoted by $\mathcal{A} \sharp \mathbb{A}$ and called the L-R-smash product of $\mathcal{A}$ and $\mathbb{A}$. In particular, for $\mathbb{A}=H$, the multiplication of $\mathcal{A} \sharp H$ is defined by

$$
(\varphi \curvearrowleft h)\left(\varphi^{\prime} \natural h^{\prime}\right)=\left(\varphi \cdot h_{2}^{\prime}\right)\left(h_{1} \cdot \varphi^{\prime}\right) \natural h_{2} h_{1}^{\prime}, \quad \forall \varphi, \varphi^{\prime} \in \mathcal{A}, h, h^{\prime} \in H .
$$




\section{The definition of the L-R-twisted tensor product of algebras}

Proposition 2.1 Let $A$ and $B$ be two (associative unital) algebras, and $R: B \otimes A \rightarrow A \otimes B$, $Q: A \otimes B \rightarrow A \otimes B$ two linear maps, with notation $R(b \otimes a)=a_{R} \otimes b_{R}, Q(a \otimes b)=a_{Q} \otimes b_{Q}$, for all $a \in A, b \in B$, satisfying the following conditions:

$$
\begin{aligned}
& a_{R} \otimes 1_{R}=a \otimes 1, \quad 1_{R} \otimes b_{R}=1 \otimes b, \\
& \left(a a^{\prime}\right)_{R} \otimes b_{R}=a_{R} a_{r}^{\prime} \otimes b_{R_{r}}, \\
& a_{R} \otimes\left(b b^{\prime}\right)_{R}=a_{R_{r}} \otimes b_{r} b_{R}^{\prime}, \\
& a_{Q} \otimes 1_{Q}=a \otimes 1, \quad 1_{Q} \otimes b_{Q}=1 \otimes b, \\
& \left(a a^{\prime}\right)_{Q} \otimes b_{Q}=a_{q} a_{Q}^{\prime} \otimes b_{Q_{q}}, \\
& a_{Q} \otimes\left(b b^{\prime}\right)_{Q}=a_{Q_{q}} \otimes b_{Q} b_{q}^{\prime}, \\
& b_{R} \otimes a_{R_{Q}} \otimes b_{Q}^{\prime}=b_{R} \otimes a_{Q_{R}} \otimes b_{Q}^{\prime}, \\
& a_{R} \otimes b_{R_{Q}} \otimes a_{Q}^{\prime}=a_{R} \otimes b_{Q_{R}} \otimes a_{Q}^{\prime},
\end{aligned}
$$

for all $a, a^{\prime} \in A$ and $b, b^{\prime} \in B$, where $r$ (respectively $q$ ) is another copy of $R$ (respectively $Q$ ). If we define on $A \otimes B$ a multiplication by $(a \otimes b)\left(a^{\prime} \otimes b^{\prime}\right)=a_{Q} a_{R}^{\prime} \otimes b_{R} b_{Q}^{\prime}$, then this multiplication is associative and $1 \otimes 1$ is the unit. This algebra structure will be denoted by $A_{Q} \otimes_{R} B$ and will be called the L-R-twisted tensor product of $A$ and $B$ afforded by the maps $R$ and $Q$.

Proof. The fact that $1 \otimes 1$ is the unit is obvious using (2.1) and (2.4), so we only prove the associativity. We compute (where $R=r=\mathcal{R}$ and $Q=q=\tilde{Q}$ ):

$$
\begin{array}{rll}
{\left[(a \otimes b)\left(a^{\prime} \otimes b^{\prime}\right)\right]\left(a^{\prime \prime} \otimes b^{\prime \prime}\right)} & = & \left(a_{Q} a_{R}^{\prime} \otimes b_{R} b_{Q}^{\prime}\right)\left(a^{\prime \prime} \otimes b^{\prime \prime}\right) \\
= & \left(a_{Q} a_{R}^{\prime}\right)_{q} a_{r}^{\prime \prime} \otimes\left(b_{R} b_{Q}^{\prime}\right)_{r} b_{q}^{\prime \prime} \\
& \stackrel{2.5}{=} & a_{Q_{q}} a_{R_{\tilde{Q}}}^{\prime} a_{r}^{\prime \prime} \otimes\left(b_{R} b_{Q}^{\prime}\right)_{r} b_{\tilde{Q}_{q}}^{\prime \prime} \\
& a_{Q_{q}} a_{R_{\tilde{Q}}}^{\prime} a_{\mathcal{R}_{r}}^{\prime \prime} \otimes b_{R_{r}} b_{Q_{\mathcal{R}}}^{\prime} b_{\tilde{Q}_{q}}^{\prime \prime} \\
\stackrel{2.3}{=} & a_{Q_{q}} a_{\tilde{Q}_{R}}^{\prime} a_{\mathcal{R}_{r}}^{\prime \prime} \otimes b_{R_{r}} b_{\mathcal{R}_{Q}}^{\prime} b_{\tilde{Q}_{q}}^{\prime \prime}, \\
& \\
& = & (a \otimes b)\left(a_{Q}^{\prime} a_{R}^{\prime \prime} \otimes b_{R}^{\prime} b_{Q}^{\prime \prime}\right) \\
& = & a_{q}\left(a_{Q}^{\prime} a_{R}^{\prime \prime}\right)_{r} \otimes b_{r}\left(b_{R}^{\prime} b_{Q}^{\prime \prime}\right)_{q} \\
& \stackrel{2.2}{=} & a_{q} a_{Q_{\mathcal{R}}}^{\prime} a_{R_{r}}^{\prime \prime} \otimes b_{\mathcal{R}_{r}}\left(b_{R}^{\prime} b_{Q}^{\prime \prime}\right)_{q} \\
& \stackrel{2.6}{=} & a_{\tilde{Q}_{q}} a_{Q_{\mathcal{R}}}^{\prime} a_{R_{r}}^{\prime \prime} \otimes b_{\mathcal{R}_{r}} b_{R_{\tilde{Q}}}^{\prime} b_{Q_{q}}^{\prime \prime},
\end{array}
$$

and we see that the two terms are equal.

Remark 2.2 If $Q=i d_{A \otimes B}$, then $A_{Q} \otimes_{R} B$ is the ordinary twisted tensor product of algebras $A \otimes_{R} B$.

Example 2.3 If $H$ is a bialgebra, $\mathcal{A}$ is an $H$-bimodule algebra and $\mathbb{A}$ is an $H$-bicomodule algebra, with notation as before, define the maps

$$
R: \mathbb{A} \otimes \mathcal{A} \rightarrow \mathcal{A} \otimes \mathbb{A}, \quad R(u \otimes \varphi)=u_{[-1]} \cdot \varphi \otimes u_{[0]},
$$




$$
Q: \mathcal{A} \otimes \mathbb{A} \rightarrow \mathcal{A} \otimes \mathbb{A}, \quad Q(\varphi \otimes u)=\varphi \cdot u_{<1>} \otimes u_{<0>}
$$

for all $\varphi \in \mathcal{A}$ and $u \in \mathbb{A}$. Then one checks that the maps $R$ and $Q$ satisfy the axioms in Proposition 2.1 and the L-R-twisted tensor product $\mathcal{A}{ }_{Q} \otimes_{R} \mathbb{A}$ coincides with the $L$ - $R$-smash product $\mathcal{A} \sqcup \mathbb{A}$. In particular, for $\mathbb{A}=H$, the above maps are given, for all $\varphi \in \mathcal{A}, h \in H$, by

$$
\begin{array}{ll}
R: H \otimes \mathcal{A} \rightarrow \mathcal{A} \otimes H, & R(h \otimes \varphi)=h_{1} \cdot \varphi \otimes h_{2}, \\
Q: \mathcal{A} \otimes H \rightarrow \mathcal{A} \otimes H, & Q(\varphi \otimes h)=\varphi \cdot h_{2} \otimes h_{1} .
\end{array}
$$

A particular case of Proposition 2.1 is obtained if $R$ is the flip map $b \otimes a \mapsto a \otimes b$ :

Corollary 2.4 Let $A, B$ be two algebras and $Q: A \otimes B \rightarrow A \otimes B$ a linear map satisfying the conditions (2.4), (2.5) and (2.6). Then the multiplication $(a \otimes b)\left(a^{\prime} \otimes b^{\prime}\right)=a_{Q} a^{\prime} \otimes b b_{Q}^{\prime}$ defines an associative algebra structure on $A \otimes B$ with unit $1 \otimes 1$, denoted by $A_{Q} \otimes B$.

Remark 2.5 Let $A, B$ be two algebras and $Q: A \otimes B \rightarrow A \otimes B$ a linear map, with notation $Q(a \otimes b)=a_{Q} \otimes b_{Q}$. Define the map $Q^{o p}: B \otimes A \rightarrow A \otimes B, Q^{o p}(b \otimes a)=a_{Q} \otimes b_{Q}$. Then one can easily check that $Q$ satisfies the conditions (2.4), (2.5) and (2.6) if and only if $Q^{\text {op }}$ is a twisting map between the opposite algebras $A^{\text {op }}$ and $B^{\text {op }}$ and in this case we have an algebra isomorphism $A_{Q} \otimes B \equiv\left(A^{o p} \otimes_{Q^{o p}} B^{o p}\right)^{o p}$, given by the trivial identification.

Remark 2.6 If $A_{Q} \otimes_{R} B$ is an L-R-twisted tensor product of algebras, we can consider also the algebras $A \otimes_{R} B$ and $A_{Q} \otimes B$.

We recall the following concept and result from [13]:

Proposition 2.7 Let $D$ be an algebra with multiplication denoted by $\mu_{D}=\mu$ and $T: D \otimes D \rightarrow$ $D \otimes D$ a linear map satisfying the following conditions: $T(1 \otimes d)=1 \otimes d, T(d \otimes 1)=d \otimes 1$, for all $d \in D$, and

$$
\begin{aligned}
& \mu_{23} \circ T_{13} \circ T_{12}=T \circ \mu_{23}: D \otimes D \otimes D \rightarrow D \otimes D, \\
& \mu_{12} \circ T_{13} \circ T_{23}=T \circ \mu_{12}: D \otimes D \otimes D \rightarrow D \otimes D, \\
& T_{12} \circ T_{23}=T_{23} \circ T_{12}: D \otimes D \otimes D \rightarrow D \otimes D \otimes D,
\end{aligned}
$$

with standard notation for $T_{i j}$ and $\mu_{i j}$. Then the bilinear map $\mu \circ T: D \otimes D \rightarrow D$ is another associative algebra structure on $D$ (with the same unit 1 ) denoted by $D^{T}$, and the map $T$ is called a twistor for $D$.

Proposition 2.8 Let $A_{Q} \otimes_{R} B$ be an L-R-twisted tensor product of algebras. Define the maps $T_{1}, T_{2}, T_{3}:(A \otimes B) \otimes(A \otimes B) \rightarrow(A \otimes B) \otimes(A \otimes B)$ by

$$
\begin{aligned}
& T_{1}\left((a \otimes b) \otimes\left(a^{\prime} \otimes b^{\prime}\right)\right)=\left(a_{Q} \otimes b_{R}\right) \otimes\left(a_{R}^{\prime} \otimes b_{Q}^{\prime}\right), \\
& T_{2}\left((a \otimes b) \otimes\left(a^{\prime} \otimes b^{\prime}\right)\right)=\left(a_{Q} \otimes b\right) \otimes\left(a^{\prime} \otimes b_{Q}^{\prime}\right), \\
& T_{3}\left((a \otimes b) \otimes\left(a^{\prime} \otimes b^{\prime}\right)\right)=\left(a \otimes b_{R}\right) \otimes\left(a_{R}^{\prime} \otimes b^{\prime}\right) .
\end{aligned}
$$

Then $T_{1}$ is a twistor for $A \otimes B, T_{2}$ is a twistor for $A \otimes_{R} B, T_{3}$ is a twistor for $A_{Q} \otimes B$ and moreover we have $A_{Q} \otimes_{R} B=(A \otimes B)^{T_{1}}=\left(A \otimes_{R} B\right)^{T_{2}}=\left(A_{Q} \otimes B\right)^{T_{3}}$.

Proof. Straightforward computations, using the relations (2.1)-(2.6). 
Proposition 2.9 Let $A \otimes_{Q} B$ be an L-R-twisted tensor product of algebras such that $Q$ is bijective with inverse $Q^{-1}$. Then the map $P: B \otimes A \rightarrow A \otimes B, P=Q^{-1} \circ R$, is a twisting map, and we have an algebra isomorphism $Q: A \otimes_{P} B \rightarrow A_{Q} \otimes_{R} B$. Thus, an L-R-twisted tensor product with bijective $Q$ is isomorphic to an ordinary twisted tensor product.

Proof. First, it is obvious that $a_{P} \otimes 1=a \otimes 1$ and $1_{P} \otimes b_{P}=1 \otimes b$, for all $a \in A, b \in B$. We check now that $P$ is a twisting map. Let $a, a^{\prime} \in A, b, b^{\prime} \in B$; we denote $Q^{-1}(a \otimes b)=a_{Q^{-1}} \otimes b_{Q^{-1}}=$ $a_{q^{-1}} \otimes b_{q^{-1}}$ and $P(b \otimes a)=a_{P} \otimes b_{P}=a_{p} \otimes b_{p}$. We compute (denote $\left.Q=q=\bar{Q}=\bar{q}\right)$ :

$$
\begin{aligned}
Q\left(a_{P} a_{p}^{\prime} \otimes b_{P_{p}}\right) & =\quad\left(a_{R_{Q^{-1}}} a_{r_{q^{-1}}}^{\prime}\right)_{Q} \otimes\left(b_{R_{Q_{q^{-1}}^{-1}}}\right)_{Q} \\
& \stackrel{(2.5)}{=} a_{R_{Q_{Q}^{-1}}} a_{r_{q-1}}^{\prime} \otimes b_{R_{Q_{r}^{-1}}^{-1}} \\
= & a_{R_{Q_{Q}^{-1}}} a_{r}^{\prime} \otimes b_{R_{Q_{r_{Q}}^{-1}}} \\
& \stackrel{(2.8)}{=} a_{R_{Q_{Q}^{-1}}} a_{r}^{\prime} \otimes b_{R_{Q_{Q_{r}}^{-1}}} \\
= & a_{R} a_{r}^{\prime} \otimes b_{R_{r}} \\
& \left(a a^{\prime}\right)_{R} \otimes b_{R},
\end{aligned}
$$

hence by applying $Q^{-1}$ we obtain $\left(a a^{\prime}\right)_{P} \otimes b_{P}=a_{P} a_{p}^{\prime} \otimes b_{P_{p}}$, that is (2.2) for $P$. The fact that $P$ satisfies (2.3) can be proved similarly. The only thing left to prove is that $Q$ is an algebra map. We compute:

$$
\begin{aligned}
& Q\left((a \otimes b)\left(a^{\prime} \otimes b^{\prime}\right)\right)=\left(a a_{P}^{\prime}\right)_{Q} \otimes\left(b_{P} b^{\prime}\right)_{Q} \\
& \stackrel{2.5}{=} a_{q} a_{P_{Q}}^{\prime} \otimes\left(b_{P} b^{\prime}\right)_{Q_{q}} \\
& \stackrel{(2.6)}{=} a_{q} a_{P_{Q_{\bar{Q}}}}^{\prime} \otimes\left(b_{P_{Q}} b_{\bar{Q}}^{\prime}\right)_{q} \\
& \stackrel{2.6)}{=} a_{q_{\bar{q}}} a_{P_{Q_{\bar{Q}}}}^{\prime} \otimes b_{P_{Q_{q}}} b_{\bar{Q}_{\bar{q}}^{\prime}} \\
& =a_{q_{\bar{q}}} a_{R_{\bar{Q}}}^{\prime} \otimes b_{R_{q}} b_{\bar{Q}_{\bar{q}}}^{\prime} \\
& \stackrel{2.7}{=} a_{q_{\bar{q}}} a_{\bar{Q}_{R}}^{\prime} \otimes b_{R_{q}} b_{\bar{Q}_{\bar{q}}}^{\prime} \\
& \stackrel{(2.8)}{=} a_{q_{\bar{q}}} a_{\bar{Q}_{R}}^{\prime} \otimes b_{q_{R}} b_{\bar{Q}_{\bar{q}}}^{\prime} \\
& =\left(a_{q} \otimes b_{q}\right)\left(a_{\bar{Q}}^{\prime} \otimes b_{\bar{Q}}^{\prime}\right) \\
& =Q(a \otimes b) Q\left(a^{\prime} \otimes b^{\prime}\right) \text {, }
\end{aligned}
$$

finishing the proof.

Remark 2.10 Proposition [2.9 generalizes (and was inspired by) the following result in [15]. Let $H$ be a Hopf algebra with bijective antipode $S$ and $\mathcal{A}$ an $H$-bimodule algebra. Consider the so-called diagonal crossed product (cf. [7], [10]) $\mathcal{A} \bowtie H$, which is an associative algebra built on $\mathcal{A} \otimes H$, with multiplication defined by $(\varphi \bowtie h)\left(\varphi^{\prime} \bowtie h^{\prime}\right)=\varphi\left(h_{1} \cdot \varphi^{\prime} \cdot S^{-1}\left(h_{3}\right)\right) \bowtie h_{2} h^{\prime}$, for all $\varphi, \varphi^{\prime} \in \mathcal{A}$ and $h, h^{\prime} \in H$, that is, $\mathcal{A} \bowtie H$ is the twisted tensor product $\mathcal{A} \otimes_{P} H$, where $P: H \otimes \mathcal{A} \rightarrow \mathcal{A} \otimes H, P(h \otimes \varphi)=h_{1} \cdot \varphi \cdot S^{-1}\left(h_{3}\right) \otimes h_{2}$. Then the map $Q: \mathcal{A} \bowtie H \rightarrow \mathcal{A} \boxminus H$, $Q(\varphi \bowtie h)=\varphi \cdot h_{2} \curvearrowleft h_{1}$, is an algebra isomorphism. 
The next result generalizes the corresponding one for twisted tensor products (cf. [5]):

Proposition 2.11 Let $A_{Q} \otimes_{R} B$ and $A^{\prime}{ }_{Q^{\prime}} \otimes_{R^{\prime}} B^{\prime}$ be two L-R-twisted tensor products of algebras and $f: A \rightarrow A^{\prime}$ and $g: B \rightarrow B^{\prime}$ two algebra maps satisfying the conditions $(f \otimes g) \circ R=R^{\prime} \circ(g \otimes f)$ and $(f \otimes g) \circ Q=Q^{\prime} \circ(f \otimes g)$. Then $f \otimes g$ is an algebra map from $A_{Q} \otimes_{R} B$ to $A^{\prime} Q_{Q^{\prime}} \otimes_{R^{\prime}} B^{\prime}$.

Proof. It is obvious that $f \otimes g$ is unital, so we only prove that it is multiplicative. Let $a, x \in A$ and $b, y \in B$; we compute:

$$
\begin{aligned}
(f \otimes g)((a \otimes b)(x \otimes y)) & =(f \otimes g)\left(a_{Q} x_{R} \otimes b_{R} y_{Q}\right) \\
& =f\left(a_{Q}\right) f\left(x_{R}\right) \otimes g\left(b_{R}\right) g\left(y_{Q}\right) \\
& =f(a)_{Q^{\prime}} f(x)_{R^{\prime}} \otimes g(b)_{R^{\prime}} g(y)_{Q^{\prime}} \\
& =(f(a) \otimes g(b))(f(x) \otimes g(y)) \\
& =(f \otimes g)(a \otimes b)(f \otimes g)(x \otimes y),
\end{aligned}
$$

finishing the proof.

\section{Iterated L-R-twisted tensor products of algebras}

It was proved in [1] that, under certain circumstances, twisted tensor products of algebras may be iterated. More precisely:

Theorem 3.1 ([11]) Let $A \otimes_{R_{1}} B, B \otimes_{R_{2}} C$ and $A \otimes_{R_{3}} C$ be twisted tensor products of algebras. Define the maps

$$
\begin{array}{ll}
T_{1}: C \otimes\left(A \otimes_{R_{1}} B\right) \rightarrow\left(A \otimes_{R_{1}} B\right) \otimes C, & T_{1}=\left(i d_{A} \otimes R_{2}\right) \circ\left(R_{3} \otimes i d_{B}\right), \\
T_{2}:\left(B \otimes_{R_{2}} C\right) \otimes A \rightarrow A \otimes\left(B \otimes_{R_{2}} C\right), & T_{2}=\left(R_{1} \otimes i d_{C}\right) \circ\left(i d_{B} \otimes R_{3}\right),
\end{array}
$$

and assume that $R_{1}, R_{2}, R_{3}$ satisfy the following compatibility condition (the hexagon equation):

$$
\left(i d_{A} \otimes R_{2}\right) \circ\left(R_{3} \otimes i d_{B}\right) \circ\left(i d_{C} \otimes R_{1}\right)=\left(R_{1} \otimes i d_{C}\right) \circ\left(i d_{B} \otimes R_{3}\right) \circ\left(R_{2} \otimes i d_{A}\right) .
$$

Then $T_{1}$ is a twisting map between $A \otimes_{R_{1}} B$ and $C, T_{2}$ is a twisting map between $A$ and $B \otimes_{R_{2}} C$ and moreover the algebras $\left(A \otimes_{R_{1}} B\right) \otimes_{T_{1}} C$ and $A \otimes_{T_{2}}\left(B \otimes_{R_{2}} C\right)$ coincide.

Our aim is now is to generalize this result for L-R-twisted tensor products of algebras. We begin with what will be our guiding example, namely a situation when L-R-smash products may be iterated.

We recall the following construction introduced in [16]. Let $H$ be a bialgebra and denote by $\mathcal{L} \mathcal{R}(H)$ the category whose objects are vector spaces $M$ endowed with $H$-bimodule and $H$-bicomodule structures (denoted by $h \otimes m \mapsto h \cdot m, m \otimes h \mapsto m \cdot h, m \mapsto m^{(-1)} \otimes m^{(0)}$, $m \mapsto m^{<0>} \otimes m^{<1>}$, for all $\left.h \in H, m \in M\right)$, such that $M$ is a left-left Yetter-Drinfeld module, a left-right Long module, a right-right Yetter-Drinfeld module and a right-left Long module, i.e.

$$
\begin{aligned}
& \left(h_{1} \cdot m\right)^{(-1)} h_{2} \otimes\left(h_{1} \cdot m\right)^{(0)}=h_{1} m^{(-1)} \otimes h_{2} \cdot m^{(0)}, \\
& (h \cdot m)^{<0>} \otimes(h \cdot m)^{<1>}=h \cdot m^{<0>} \otimes m^{<1>}, \\
& \left(m \cdot h_{2}\right)^{<0>} \otimes h_{1}\left(m \cdot h_{2}\right)^{<1>}=m^{<0>} \cdot h_{1} \otimes m^{<1>} h_{2}, \\
& (m \cdot h)^{(-1)} \otimes(m \cdot h)^{(0)}=m^{(-1)} \otimes m^{(0)} \cdot h,
\end{aligned}
$$


for all $h \in H, m \in M$; the morphisms in $\mathcal{L R}(H)$ are the $H$-bilinear $H$-bicolinear maps. The objects of $\mathcal{L} \mathcal{R}(H)$ are called Yetter-Drinfeld-Long bimodules. This category $\mathcal{L} \mathcal{R}(H)$ is a strict monoidal category, with unit $k$ endowed with usual $H$-bimodule and $H$-bicomodule structures, and tensor product given as follows: if $M, N \in \mathcal{L R}(H)$ then $M \otimes N \in \mathcal{L R}(H)$ with structures (for all $m \in M, n \in N, h \in H$ ):

$$
\begin{aligned}
& h \cdot(m \otimes n)=h_{1} \cdot m \otimes h_{2} \cdot n, \\
& (m \otimes n) \cdot h=m \cdot h_{1} \otimes n \cdot h_{2}, \\
& (m \otimes n)^{(-1)} \otimes(m \otimes n)^{(0)}=m^{(-1)} n^{(-1)} \otimes\left(m^{(0)} \otimes n^{(0)}\right), \\
& (m \otimes n)^{<0>} \otimes(m \otimes n)^{<1>}=\left(m^{<0>} \otimes n^{<0>}\right) \otimes m^{<1>} n^{<1>} .
\end{aligned}
$$

Let now $A$ be an algebra in the monoidal category $\mathcal{L} \mathcal{R}(H)$. By looking at the definition of $\mathcal{L} \mathcal{R}(H)$ as a monoidal category, it is easy to see that, in particular, $A$ is an $H$-bimodule algebra and an $H$-bicomodule algebra. Thus, if $\mathcal{A}$ is an $H$-bimodule algebra, we can consider the associative algebras $\mathcal{A} \natural A$ and $A \natural H$.

Proposition 3.2 Let $H$ be a bialgebra, $A$ an algebra in $\mathcal{L} \mathcal{R}(H)$ and $\mathcal{A}$ an $H$-bimodule algebra. Then:

(i) $\mathcal{A}$ $A$ is an $H$-bimodule algebra, with $H$-module structures given by $h \cdot(\varphi \curvearrowleft a)=h_{1} \cdot \varphi \natural h_{2} \cdot a$ and $(\varphi \curvearrowleft a) \cdot h=\varphi \cdot h_{2} \downarrow a \cdot h_{1}$, for all $\varphi \in \mathcal{A}, a \in A, h \in H$.

(ii) $A$ $H$ is an $H$-bicomodule algebra, with $H$-comodule structures given by

$$
\begin{aligned}
& \lambda: A \natural H \rightarrow H \otimes(A \natural H), \quad \lambda(a \natural h)=a^{(-1)} h_{1} \otimes\left(a^{(0)} \natural h_{2}\right):=(a \natural h)_{[-1]} \otimes(a \natural h)_{[0]}, \\
& \rho: A \curvearrowleft H \rightarrow(A \natural H) \otimes H, \quad \rho(a \curvearrowleft h)=\left(a^{<0>} \curvearrowleft h_{1}\right) \otimes h_{2} a^{<1>}:=(a \curvearrowleft h)_{<0>} \otimes(a \natural h)_{<1>} .
\end{aligned}
$$

(iii) The algebras $(\mathcal{A} \boxminus A) \natural H$ and $\mathcal{A} \boxminus(A \curvearrowleft H)$ coincide.

Proof. (i) Obviously, $\mathcal{A} \curvearrowleft A$ is an $H$-bimodule. Let $h \in H, \varphi, \varphi^{\prime} \in \mathcal{A}$ and $a, a^{\prime} \in A$. We check the left and right $H$-module algebra conditions:

$$
\begin{aligned}
& \left(h_{1} \cdot(\varphi \curvearrowleft a)\right)\left(h_{2} \cdot\left(\varphi^{\prime} \curvearrowleft a^{\prime}\right)\right)=\left(h_{1} \cdot \varphi \curvearrowleft h_{2} \cdot a\right)\left(h_{3} \cdot \varphi^{\prime} \curvearrowleft h_{4} \cdot a^{\prime}\right) \\
& =\left(\left(h_{1} \cdot \varphi\right) \cdot\left(h_{4} \cdot a^{\prime}\right)^{<1>}\right)\left(\left(h_{2} \cdot a\right)^{(-1)} \cdot\left(h_{3} \cdot \varphi^{\prime}\right)\right) \\
& \natural\left(h_{2} \cdot a\right)^{(0)}\left(h_{4} \cdot a^{\prime}\right)^{<0>} \\
& \stackrel{3.2}{=}\left(h_{1} \cdot \varphi \cdot a^{\prime<1>}\right)\left(\left(h_{2} \cdot a\right)^{(-1)} h_{3} \cdot \varphi^{\prime}\right) \natural\left(h_{2} \cdot a\right)^{(0)}\left(h_{4} \cdot a^{\prime<0>}\right) \\
& \stackrel{\text { 3.1 }}{=}\left(h_{1} \cdot \varphi \cdot a^{\prime<1>}\right)\left(h_{2} a^{(-1)} \cdot \varphi^{\prime}\right) \natural\left(h_{3} \cdot a^{(0)}\right)\left(h_{4} \cdot a^{\prime<0>}\right) \\
& =h \cdot\left(\left(\varphi \cdot a^{\prime<1>}\right)\left(a^{(-1)} \cdot \varphi^{\prime}\right) \natural a^{(0)} a^{\prime<0>}\right) \\
& =h \cdot\left((\varphi \curvearrowleft a)\left(\varphi^{\prime} \downarrow a^{\prime}\right)\right) \text {, q.e.d. } \\
& \left((\varphi \curvearrowleft a) \cdot h_{1}\right)\left(\left(\varphi^{\prime} \natural a^{\prime}\right) \cdot h_{2}\right)=\left(\varphi \cdot h_{2} \natural a \cdot h_{1}\right)\left(\varphi^{\prime} \cdot h_{4} \natural a^{\prime} \cdot h_{3}\right) \\
& =\left(\left(\varphi \cdot h_{2}\right) \cdot\left(a^{\prime} \cdot h_{3}\right)^{<1>}\right)\left(\left(a \cdot h_{1}\right)^{(-1)} \cdot\left(\varphi^{\prime} \cdot h_{4}\right)\right) \\
& \natural\left(a \cdot h_{1}\right)^{(0)}\left(a^{\prime} \cdot h_{3}\right)^{<0>} \\
& \stackrel{\text { (3.4) }}{=}\left(\varphi \cdot h_{2}\left(a^{\prime} \cdot h_{3}\right)^{<1>}\right)\left(a^{(-1)} \cdot \varphi^{\prime} \cdot h_{4}\right) \natural\left(a^{(0)} \cdot h_{1}\right)\left(a^{\prime} \cdot h_{3}\right)^{<0>} \\
& \stackrel{3.3}{=}\left(\varphi \cdot a^{\prime<1>} h_{3}\right)\left(a^{(-1)} \cdot \varphi^{\prime} \cdot h_{4}\right) \curvearrowleft\left(a^{(0)} \cdot h_{1}\right)\left(a^{\prime<0>} \cdot h_{2}\right) \\
& =\left(\left(\varphi \cdot a^{\prime<1>}\right)\left(a^{(-1)} \cdot \varphi^{\prime}\right)\right) \cdot h_{2} \natural\left(a^{(0)} a^{\prime<0>}\right) \cdot h_{1}
\end{aligned}
$$




$$
\begin{aligned}
& =\left(\left(\varphi \cdot a^{\prime<1>}\right)\left(a^{(-1)} \cdot \varphi^{\prime}\right) \natural a^{(0)} a^{\prime<0>}\right) \cdot h \\
& =\left((\varphi \natural a)\left(\varphi^{\prime} \natural a^{\prime}\right)\right) \cdot h, \quad \text { q.e.d. }
\end{aligned}
$$

(ii) It is very easy to see that $A \natural H$ is an $H$-bicomodule, so we check the left and right $H$ comodule algebra conditions:

$$
\begin{aligned}
& \lambda\left((a \natural h)\left(a^{\prime} \natural h^{\prime}\right)\right)=\lambda\left(\left(a \cdot h_{2}^{\prime}\right)\left(h_{1} \cdot a^{\prime}\right) \natural h_{2} h_{1}^{\prime}\right) \\
& =\left(a \cdot h_{3}^{\prime}\right)^{(-1)}\left(h_{1} \cdot a^{\prime}\right)^{(-1)} h_{2} h_{1}^{\prime} \otimes\left(\left(a \cdot h_{3}^{\prime}\right)^{(0)}\left(h_{1} \cdot a^{\prime}\right)^{(0)} \natural h_{3} h_{2}^{\prime}\right) \\
& \stackrel{\text { (3.4) }}{=} a^{(-1)}\left(h_{1} \cdot a^{\prime}\right)^{(-1)} h_{2} h_{1}^{\prime} \otimes\left(\left(a^{(0)} \cdot h_{3}^{\prime}\right)\left(h_{1} \cdot a^{\prime}\right)^{(0)} \natural h_{3} h_{2}^{\prime}\right) \\
& \stackrel{\text { 3.1 }}{=} a^{(-1)} h_{1} a^{(-1)} h_{1}^{\prime} \otimes\left(\left(a^{(0)} \cdot h_{3}^{\prime}\right)\left(h_{2} \cdot a^{\prime(0)}\right) \natural h_{3} h_{2}^{\prime}\right) \\
& =\left(a^{(-1)} h_{1} \otimes\left(a^{(0)} \natural h_{2}\right)\right)\left(a^{\prime(-1)} h_{1}^{\prime} \otimes\left(a^{\prime(0)} \natural h_{2}^{\prime}\right)\right) \\
& =\lambda(a \curvearrowleft h) \lambda\left(a^{\prime} \curvearrowleft h^{\prime}\right) \text {, q.e.d. } \\
& \rho\left((a \curvearrowleft h)\left(a^{\prime} \natural h^{\prime}\right)\right)=\rho\left(\left(a \cdot h_{2}^{\prime}\right)\left(h_{1} \cdot a^{\prime}\right) \natural h_{2} h_{1}^{\prime}\right) \\
& =\left(\left(a \cdot h_{3}^{\prime}\right)^{<0>}\left(h_{1} \cdot a^{\prime}\right)^{<0>} \curvearrowleft h_{2} h_{1}^{\prime}\right) \otimes h_{3} h_{2}^{\prime}\left(a \cdot h_{3}^{\prime}\right)^{<1>}\left(h_{1} \cdot a^{\prime}\right)^{<1>} \\
& \stackrel{3.2}{=}\left(\left(a \cdot h_{3}^{\prime}\right)^{<0>}\left(h_{1} \cdot a^{\prime<0>}\right) \natural h_{2} h_{1}^{\prime}\right) \otimes h_{3} h_{2}^{\prime}\left(a \cdot h_{3}^{\prime}\right)^{<1>} a^{\prime<1>} \\
& \stackrel{3.3}{=}\left(\left(a^{<0>} \cdot h_{2}^{\prime}\right)\left(h_{1} \cdot a^{\prime<0>}\right) \natural h_{2} h_{1}^{\prime}\right) \otimes h_{3} a^{<1>} h_{3}^{\prime} a^{\prime<1>} \\
& =\left(a^{<0>} \natural h_{1}\right)\left(a^{\prime<0>} \boxminus h_{1}^{\prime}\right) \otimes h_{2} a^{<1>} h_{2}^{\prime} a^{\prime<1>} \\
& =\rho(a \natural h) \rho\left(a^{\prime} \natural h^{\prime}\right) \text {, q.e.d. }
\end{aligned}
$$

(iii) We write down the multiplication of $(\mathcal{A} \curvearrowleft A) \natural H$ :

$$
\begin{aligned}
& ((\varphi \curvearrowleft a) \natural h)\left(\left(\varphi^{\prime} \natural a^{\prime}\right) \natural h^{\prime}\right)=\left((\varphi \curvearrowleft a) \cdot h_{2}^{\prime}\right)\left(h_{1} \cdot\left(\varphi^{\prime} \natural a^{\prime}\right)\right) \natural h_{2} h_{1}^{\prime} \\
& =\left(\varphi \cdot h_{3}^{\prime} \curvearrowleft a \cdot h_{2}^{\prime}\right)\left(h_{1} \cdot \varphi^{\prime} \curvearrowleft h_{2} \cdot a^{\prime}\right) \natural h_{3} h_{1}^{\prime} \\
& =\left(\varphi \cdot h_{3}^{\prime}\left(h_{2} \cdot a^{\prime}\right)^{<1>}\right)\left(\left(a \cdot h_{2}^{\prime}\right)^{(-1)} h_{1} \cdot \varphi^{\prime}\right) \\
& \natural\left(a \cdot h_{2}^{\prime}\right)^{(0)}\left(h_{2} \cdot a^{\prime}\right)^{<0>} \natural h_{3} h_{1}^{\prime} \\
& \stackrel{(3.2),(3.4)}{=}\left(\varphi \cdot h_{3}^{\prime} a^{\prime<1>}\right)\left(a^{(-1)} h_{1} \cdot \varphi^{\prime}\right) \natural\left(a^{(0)} \cdot h_{2}^{\prime}\right)\left(h_{2} \cdot a^{\prime<0>}\right) \natural h_{3} h_{1}^{\prime} \text {. }
\end{aligned}
$$

We write down the multiplication of $\mathcal{A} \natural(A \curvearrowleft H)$ :

$$
\begin{aligned}
& (\varphi \curvearrowleft(a \curvearrowleft h))\left(\varphi^{\prime} \natural\left(a^{\prime} \natural h^{\prime}\right)\right)=\left(\varphi \cdot\left(a^{\prime} \natural h^{\prime}\right)_{<1>}\right)\left((a \curvearrowleft h)_{[-1]} \cdot \varphi^{\prime}\right) \natural(a \natural h)_{[0]}\left(a^{\prime} \natural h^{\prime}\right)_{<0>} \\
& =\left(\varphi \cdot h_{2}^{\prime} a^{\prime<1>}\right)\left(a^{(-1)} h_{1} \cdot \varphi^{\prime}\right) \natural\left(a^{(0)} \natural h_{2}\right)\left(a^{\prime<0>} \boxminus h_{1}^{\prime}\right) \\
& =\left(\varphi \cdot h_{3}^{\prime} a^{\prime<1>}\right)\left(a^{(-1)} h_{1} \cdot \varphi^{\prime}\right) \downarrow\left(a^{(0)} \cdot h_{2}^{\prime}\right)\left(h_{2} \cdot a^{\prime<0>}\right) \natural h_{3} h_{1}^{\prime} \text {, }
\end{aligned}
$$

and we see that the two multiplications coincide.

We are able now to find a common generalization of Theorem 3.1 and Proposition 3.2

Theorem 3.3 Let $A_{Q_{1}} \otimes_{R_{1}} B, B Q_{Q_{2}} \otimes_{R_{2}} C, A_{Q_{3}} \otimes_{R_{3}} C$ be three L-R-twisted tensor products of algebras, such that the following conditions are satisfied, for all $a \in A, b \in B, c \in C$ :

$$
\begin{aligned}
& \left(a_{R_{1}}\right)_{R_{3}} \otimes\left(b_{R_{1}}\right)_{R_{2}} \otimes\left(c_{R_{3}}\right)_{R_{2}}=\left(a_{R_{3}}\right)_{R_{1}} \otimes\left(b_{R_{2}}\right)_{R_{1}} \otimes\left(c_{R_{2}}\right)_{R_{3}}, \\
& \left(a_{Q_{1}}\right)_{Q_{3}} \otimes\left(b_{Q_{1}}\right)_{Q_{2}} \otimes\left(c_{Q_{3}}\right)_{Q_{2}}=\left(a_{Q_{3}}\right)_{Q_{1}} \otimes\left(b_{Q_{2}}\right)_{Q_{1}} \otimes\left(c_{Q_{2}}\right)_{Q_{3}},
\end{aligned}
$$




$$
\begin{aligned}
& a_{R_{1}} \otimes\left(b_{R_{1}}\right)_{Q_{2}} \otimes c_{Q_{2}}=a_{R_{1}} \otimes\left(b_{Q_{2}}\right)_{R_{1}} \otimes c_{Q_{2}}, \\
& a_{Q_{1}} \otimes\left(b_{R_{2}}\right)_{Q_{1}} \otimes c_{R_{2}}=a_{Q_{1}} \otimes\left(b_{Q_{1}}\right)_{R_{2}} \otimes c_{R_{2}}, \\
& \left(a_{Q_{1}}\right)_{R_{3}} \otimes b_{Q_{1}} \otimes c_{R_{3}}=\left(a_{R_{3}}\right)_{Q_{1}} \otimes b_{Q_{1}} \otimes c_{R_{3}}, \\
& \left(a_{R_{1}}\right)_{Q_{3}} \otimes b_{R_{1}} \otimes c_{Q_{3}}=\left(a_{Q_{3}}\right)_{R_{1}} \otimes b_{R_{1}} \otimes c_{Q_{3}}, \\
& a_{R_{3}} \otimes b_{Q_{2}} \otimes\left(c_{Q_{2}}\right)_{R_{3}}=a_{R_{3}} \otimes b_{Q_{2}} \otimes\left(c_{R_{3}}\right)_{Q_{2}}, \\
& a_{Q_{3}} \otimes b_{R_{2}} \otimes\left(c_{Q_{3}}\right)_{R_{2}}=a_{Q_{3}} \otimes b_{R_{2}} \otimes\left(c_{R_{2}}\right)_{Q_{3}} .
\end{aligned}
$$

Define the maps

$$
\begin{array}{ll}
T_{1}: C \otimes(A \otimes B) \rightarrow(A \otimes B) \otimes C, & T_{1}(c \otimes(a \otimes b))=\left(a_{R_{3}} \otimes b_{R_{2}}\right) \otimes\left(c_{R_{3}}\right)_{R_{2}}, \\
V_{1}:(A \otimes B) \otimes C \rightarrow(A \otimes B) \otimes C, & V_{1}((a \otimes b) \otimes c)=\left(a_{Q_{3}} \otimes b_{Q_{2}}\right) \otimes\left(c_{Q_{3}}\right)_{Q_{2}}, \\
T_{2}:(B \otimes C) \otimes A \rightarrow A \otimes(B \otimes C), & T_{2}((b \otimes c) \otimes a)=\left(a_{R_{3}}\right)_{R_{1}} \otimes\left(b_{R_{1}} \otimes c_{R_{3}}\right), \\
V_{2}: A \otimes(B \otimes C) \rightarrow A \otimes(B \otimes C), & V_{2}(a \otimes(b \otimes c))=\left(a_{Q_{3}}\right)_{Q_{1}} \otimes\left(b_{Q_{1}} \otimes c_{Q_{3}}\right) .
\end{array}
$$

Then $\left(A_{Q_{1}} \otimes_{R_{1}} B\right)_{V_{1}} \otimes_{T_{1}} C$ and $A_{V_{2}} \otimes_{T_{2}}\left(B_{Q_{2}} \otimes_{R_{2}} C\right)$ are L-R-twisted tensor products of algebras and moreover they coincide as algebras.

Proof. We only give the proof for $\left(A_{Q_{1}} \otimes_{R_{1}} B\right)_{V_{1}} \otimes_{T_{1}} C$, the one for $A_{V_{2}} \otimes_{T_{2}}\left(B_{Q_{2}} \otimes_{R_{2}} C\right)$ is similar and left to the reader. We need to prove the relations (2.1)-(2.8) for the maps $T_{1}$ and $V_{1}$. We only prove (2.2), (2.5) and (2.8), the other relations are very easy to prove and are left to the reader.

Proof of (2.2):

$$
\begin{array}{rll}
\left((a \otimes b)\left(a^{\prime} \otimes b^{\prime}\right)\right)_{T_{1}} \otimes c_{T_{1}} & = & \left(a_{Q_{1}} a_{R_{1}}^{\prime} \otimes b_{R_{1}} b_{Q_{1}}^{\prime}\right)_{T_{1}} \otimes c_{T_{1}} \\
& = & \left(a_{Q_{1}} a_{R_{1}}^{\prime}\right)_{R_{3}} \otimes\left(b_{R_{1}} b_{Q_{1}}^{\prime}\right)_{R_{2}} \otimes\left(c_{R_{3}}\right)_{R_{2}} \\
& \stackrel{\sqrt{2.2})}{=} & \left(a_{Q_{1}}\right)_{R_{3}}\left(a_{R_{1}}^{\prime}\right)_{r_{3}} \otimes\left(b_{R_{1}} b_{Q_{1}}^{\prime}\right)_{R_{2}} \otimes\left(\left(c_{R_{3}}\right)_{r_{3}}\right)_{R_{2}} \\
& \stackrel{\sqrt{2.2})}{=} & \left(a_{Q_{1}}\right)_{R_{3}}\left(a_{R_{1}}^{\prime}\right)_{r_{3}} \otimes\left(b_{R_{1}}\right)_{R_{2}}\left(b_{Q_{1}}^{\prime}\right)_{r_{2}} \otimes\left(\left(\left(c_{R_{3}}\right)_{r_{3}}\right)_{R_{2}}\right)_{r_{2}} \\
& \stackrel{3.8}{=} & \left(a_{Q_{1}}\right)_{R_{3}}\left(a_{R_{1}}^{\prime}\right)_{r_{3}} \otimes\left(b_{R_{1}}\right)_{R_{2}}\left(b_{r_{2}}^{\prime}\right)_{Q_{1}} \otimes\left(\left(\left(c_{R_{3}}\right)_{r_{3}}\right)_{R_{2}}\right)_{r_{2}} \\
& \stackrel{3.9}{=} & \left(a_{R_{3}}\right)_{Q_{1}}\left(a_{R_{1}}^{\prime}\right)_{r_{3}} \otimes\left(b_{R_{1}}\right)_{R_{2}}\left(b_{r_{2}}^{\prime}\right)_{Q_{1}} \otimes\left(\left(\left(c_{R_{3}}\right)_{r_{3}}\right)_{R_{2}}\right)_{r_{2}} \\
& \stackrel{3.5}{=} & \left(a_{R_{3}}\right)_{Q_{1}}\left(a_{r_{3}}^{\prime}\right)_{R_{1}} \otimes\left(b_{R_{2}}\right)_{R_{1}}\left(b_{r_{2}}^{\prime}\right)_{Q_{1}} \otimes\left(\left(\left(c_{R_{3}}\right)_{R_{2}}\right)_{r_{3}}\right)_{r_{2}} \\
= & \left(a_{R_{3}} \otimes b_{R_{2}}\right)\left(a_{r_{3}}^{\prime} \otimes b_{r_{2}}^{\prime}\right) \otimes\left(\left(\left(c_{R_{3}}\right)_{R_{2}}\right)_{r_{3}}\right)_{r_{2}} \\
= & (a \otimes b)_{T_{1}}\left(a^{\prime} \otimes b^{\prime}\right)_{t_{1}} \otimes\left(c_{T_{1}}\right)_{t_{1}}, \quad \text { q.e.d. }
\end{array}
$$

Proof of (2.5):

$$
\begin{array}{rll}
\left((a \otimes b)\left(a^{\prime} \otimes b^{\prime}\right)\right)_{V_{1}} \otimes c_{V_{1}} & = & \left(a_{Q_{1}} a_{R_{1}}^{\prime} \otimes b_{R_{1}} b_{Q_{1}}^{\prime}\right)_{V_{1}} \otimes c_{V_{1}} \\
& = & \left(a_{Q_{1}} a_{R_{1}}^{\prime}\right)_{Q_{3}} \otimes\left(b_{R_{1}} b_{Q_{1}}^{\prime}\right)_{Q_{2}} \otimes\left(c_{Q_{3}}\right)_{Q_{2}} \\
& \stackrel{(2.5)}{=} & \left(a_{Q_{1}}\right)_{q_{3}}\left(a_{R_{1}}^{\prime}\right)_{Q_{3}} \otimes\left(b_{R_{1}} b_{Q_{1}}^{\prime}\right)_{Q_{2}} \otimes\left(\left(c_{Q_{3}}\right)_{q_{3}}\right)_{Q_{2}} \\
& \left(a_{Q_{1}}\right)_{q_{3}}\left(a_{R_{1}}^{\prime}\right)_{Q_{3}} \otimes\left(b_{R_{1}}\right)_{q_{2}}\left(b_{Q_{1}}^{\prime}\right)_{Q_{2}} \otimes\left(\left(\left(c_{Q_{3}}\right)_{q_{3}}\right)_{Q_{2}}\right)_{q_{2}} \\
& \stackrel{2.5}{=} & \left(a_{q_{3}}\right)_{Q_{1}}\left(a_{R_{1}}^{\prime}\right)_{Q_{3}} \otimes\left(b_{R_{1}}\right)_{q_{2}}\left(b_{Q_{2}}^{\prime}\right)_{Q_{1}} \otimes\left(\left(\left(c_{Q_{3}}\right)_{Q_{2}}\right)_{q_{3}}\right)_{q_{2}}
\end{array}
$$




$$
\begin{array}{ll}
\stackrel{\text { 3.7) }}{=} & \left(a_{q_{3}}\right)_{Q_{1}}\left(a_{R_{1}}^{\prime}\right)_{Q_{3}} \otimes\left(b_{q_{2}}\right)_{R_{1}}\left(b_{Q_{2}}^{\prime}\right)_{Q_{1}} \otimes\left(\left(\left(c_{Q_{3}}\right)_{Q_{2}}\right)_{q_{3}}\right)_{q_{2}} \\
\stackrel{\text { 3.10) }}{=} & \left(a_{q_{3}}\right)_{Q_{1}}\left(a_{Q_{3}}^{\prime}\right)_{R_{1}} \otimes\left(b_{q_{2}}\right)_{R_{1}}\left(b_{Q_{2}}^{\prime}\right)_{Q_{1}} \otimes\left(\left(\left(c_{Q_{3}}\right)_{Q_{2}}\right)_{q_{3}}\right)_{q_{2}} \\
= & \left(a_{q_{3}} \otimes b_{q_{2}}\right)\left(a_{Q_{3}}^{\prime} \otimes b_{Q_{2}}^{\prime}\right) \otimes\left(\left(\left(c_{Q_{3}}\right)_{Q_{2}}\right)_{q_{3}}\right)_{q_{2}} \\
= & (a \otimes b)_{v_{1}}\left(a^{\prime} \otimes b^{\prime}\right)_{V_{1}} \otimes\left(c_{V_{1}}\right)_{v_{1}}, \quad \text { q.e.d. }
\end{array}
$$

Proof of (2.8):

$$
\begin{aligned}
& (a \otimes b)_{T_{1}} \otimes\left(c_{V_{1}}\right)_{T_{1}} \otimes\left(a^{\prime} \otimes b^{\prime}\right)_{V_{1}}=\left(a_{R_{3}} \otimes b_{R_{2}}\right) \otimes\left(\left(\left(c_{Q_{3}}\right)_{Q_{2}}\right)_{R_{3}}\right)_{R_{2}} \otimes\left(a_{Q_{3}}^{\prime} \otimes b_{Q_{2}}^{\prime}\right) \\
& \stackrel{3.11}{=}\left(a_{R_{3}} \otimes b_{R_{2}}\right) \otimes\left(\left(\left(c_{Q_{3}}\right)_{R_{3}}\right)_{Q_{2}}\right)_{R_{2}} \otimes\left(a_{Q_{3}}^{\prime} \otimes b_{Q_{2}}^{\prime}\right) \\
& \stackrel{2.8}{=}\left(a_{R_{3}} \otimes b_{R_{2}}\right) \otimes\left(\left(\left(c_{R_{3}}\right)_{Q_{3}}\right)_{R_{2}}\right)_{Q_{2}} \otimes\left(a_{Q_{3}}^{\prime} \otimes b_{Q_{2}}^{\prime}\right) \\
& \stackrel{3.12}{=}\left(a_{R_{3}} \otimes b_{R_{2}}\right) \otimes\left(\left(\left(c_{R_{3}}\right)_{R_{2}}\right)_{Q_{3}}\right)_{Q_{2}} \otimes\left(a_{Q_{3}}^{\prime} \otimes b_{Q_{2}}^{\prime}\right) \\
& =(a \otimes b)_{T_{1}} \otimes\left(c_{T_{1}}\right)_{V_{1}} \otimes\left(a^{\prime} \otimes b^{\prime}\right)_{V_{1}} \text {, q.e.d. }
\end{aligned}
$$

We prove now that $\left(A_{Q_{1}} \otimes_{R_{1}} B\right)_{V_{1}} \otimes_{T_{1}} C \equiv A_{V_{2}} \otimes_{T_{2}}\left(B_{Q_{2}} \otimes_{R_{2}} C\right)$. We write down the multiplication of $\left(A_{Q_{1}} \otimes_{R_{1}} B\right)_{V_{1}} \otimes_{T_{1}} C$ :

$$
\begin{aligned}
& ((a \otimes b) \otimes c)\left(\left(a^{\prime} \otimes b^{\prime}\right) \otimes c^{\prime}\right) \quad=\quad(a \otimes b)_{V_{1}}\left(a^{\prime} \otimes b^{\prime}\right)_{T_{1}} \otimes c_{T_{1}} c_{V_{1}}^{\prime} \\
& =\left(a_{Q_{3}} \otimes b_{Q_{2}}\right)\left(a_{R_{3}}^{\prime} \otimes b_{R_{2}}^{\prime}\right) \otimes\left(c_{R_{3}}\right)_{R_{2}}\left(c_{Q_{3}}^{\prime}\right)_{Q_{2}} \\
& =\left(a_{Q_{3}}\right)_{Q_{1}}\left(a_{R_{3}}^{\prime}\right)_{R_{1}} \otimes\left(b_{Q_{2}}\right)_{R_{1}}\left(b_{R_{2}}^{\prime}\right)_{Q_{1}} \otimes\left(c_{R_{3}}\right)_{R_{2}}\left(c_{Q_{3}}^{\prime}\right)_{Q_{2}} \\
& \stackrel{(3.8)}{=}\left(a_{Q_{3}}\right)_{Q_{1}}\left(a_{R_{3}}^{\prime}\right)_{R_{1}} \otimes\left(b_{Q_{2}}\right)_{R_{1}}\left(b_{Q_{1}}^{\prime}\right)_{R_{2}} \otimes\left(c_{R_{3}}\right)_{R_{2}}\left(c_{Q_{3}}^{\prime}\right)_{Q_{2}} \text {. }
\end{aligned}
$$

We write down the multiplication of $A_{V_{2}} \otimes_{T_{2}}\left(B_{Q_{2}} \otimes_{R_{2}} C\right)$ :

$$
\begin{aligned}
(a \otimes(b \otimes c))\left(a^{\prime} \otimes\left(b^{\prime} \otimes c^{\prime}\right)\right) & =\quad a_{V_{2}} a_{T_{2}}^{\prime} \otimes(b \otimes c)_{T_{2}}\left(b^{\prime} \otimes c^{\prime}\right)_{V_{2}} \\
& =\left(a_{Q_{3}}\right)_{Q_{1}}\left(a_{R_{3}}^{\prime}\right)_{R_{1}} \otimes\left(b_{R_{1}} \otimes c_{R_{3}}\right)\left(b_{Q_{1}}^{\prime} \otimes c_{Q_{3}}^{\prime}\right) \\
& =\left(a_{Q_{3}}\right)_{Q_{1}}\left(a_{R_{3}}^{\prime}\right)_{R_{1}} \otimes\left(b_{R_{1}}\right)_{Q_{2}}\left(b_{Q_{1}}^{\prime}\right)_{R_{2}} \otimes\left(c_{R_{3}}\right)_{R_{2}}\left(c_{Q_{3}}^{\prime}\right)_{Q_{2}} \\
& \stackrel{\text { B.7b }}{=}\left(a_{Q_{3}}\right)_{Q_{1}}\left(a_{R_{3}}^{\prime}\right)_{R_{1}} \otimes\left(b_{Q_{2}}\right)_{R_{1}}\left(b_{Q_{1}}^{\prime}\right)_{R_{2}} \otimes\left(c_{R_{3}}\right)_{R_{2}}\left(c_{Q_{3}}^{\prime}\right)_{Q_{2}},
\end{aligned}
$$

and we can see that the two formulae are identical.

\section{Invariance under twisting}

Let $H$ be a bialgebra and $F \in H \otimes H$ a 2-cocycle, that is $F$ is invertible and satisfies

$$
\begin{aligned}
& (\varepsilon \otimes i d)(F)=(i d \otimes \varepsilon)(F)=1, \\
& (1 \otimes F)(i d \otimes \Delta)(F)=(F \otimes 1)(\Delta \otimes i d)(F) .
\end{aligned}
$$

We denote $F=F^{1} \otimes F^{2}$ and $F^{-1}=G^{1} \otimes G^{2}$. We denote by $H_{F}$ the Drinfeld twist of $H$, which is a bialgebra having the same algebra structure as $H$ and comultiplication given by $\Delta_{F}(h)=F \Delta(h) F^{-1}$, for all $h \in H$.

If $A$ is a left $H$-module algebra (with $H$-action denoted by $h \otimes a \mapsto h \cdot a$ ), the invariance under twisting of the smash product $A \# H$ is the following result (see [14, [6]). Define a new 
multiplication on $A$, by $a * a^{\prime}=\left(G^{1} \cdot a\right)\left(G^{2} \cdot a^{\prime}\right)$, for all $a, a^{\prime} \in A$, and denote by $A_{F^{-1}}$ the new structure; then $A_{F^{-1}}$ is a left $H_{F}$-module algebra (with the same action as for $A$ ) and we have an algebra isomorphism $A_{F^{-1}} \# H_{F} \simeq A \# H, a \# h \mapsto G^{1} \cdot a \# G^{2} h$.

This result was regarded in [11] as a particular case of a very general result (Theorem 4.4) for twisted tensor products of algebras, that was called "invariance under twisting" for twisted tensor products of algebras.

Let again $H$ be a bialgebra, $F \in H \otimes H$ a 2-cocycle and $\mathcal{A}$ an $H$-bimodule algebra. Define a new multiplication on $\mathcal{A}$, by $\varphi \bullet \varphi^{\prime}=\left(G^{1} \cdot \varphi \cdot F^{1}\right)\left(G^{2} \cdot \varphi^{\prime} \cdot F^{2}\right)$, where $F=F^{1} \otimes F^{2}$ and $F^{-1}=G^{1} \otimes G^{2}$, and denote by ${ }_{F} \mathcal{A}_{F^{-1}}$ the new structure. Then one can easily see that ${ }_{F} \mathcal{A}_{F^{-1}}$ is an $H_{F}$-bimodule algebra, and moreover we have the following invariance under twisting for L-R-smash products:

Proposition 4.1 We have an algebra isomorphism

$$
{ }_{F} \mathcal{A}_{F^{-1}} \natural H_{F} \simeq \mathcal{A} \natural H, \quad \varphi \natural h \mapsto G^{1} \cdot \varphi \cdot F^{2} \natural G^{2} h F^{1} .
$$

Our aim is to prove an "invariance under twisting" for L-R-twisted tensor products of algebras, that is, to find a common generalization of Proposition 4.1 and of Theorem 4.4 in [11].

Proposition 4.2 Let $A, B$ be two algebras and $R: B \otimes A \rightarrow A \otimes B, Q: A \otimes B \rightarrow A \otimes B$ two linear maps, with notation $R(b \otimes a)=a_{R} \otimes b_{R}$ and $Q(a \otimes b)=a_{Q} \otimes b_{Q}$, for all $a \in A$ and $b \in B$, such that (2.7) holds. Assume that we are given linear maps $\mu_{l}: B \otimes A \rightarrow A$, $b \otimes a \mapsto b \cdot a, \mu_{r}: A \otimes B \rightarrow A, a \otimes b \mapsto a \cdot b, \rho_{r}: A \rightarrow A \otimes B, \rho_{r}(a)=a_{(0)} \otimes a_{(1)}, \rho_{l}: A \rightarrow B \otimes A$, $\rho_{l}(a)=a_{<-1>} \otimes a_{<0>}$, and denote $a \bullet a^{\prime}:=\left(a_{(0)} \cdot a_{<-1>}^{\prime}\right)\left(a_{(1)} \cdot a_{<0>}^{\prime}\right)$, for all $a, a^{\prime} \in A$. Assume that the following conditions are satisfied: $\rho_{r}(1)=1 \otimes 1, \rho_{l}(1)=1 \otimes 1,1 \cdot a=a=a \cdot 1$, $a_{(0)}\left(a_{(1)} \cdot 1\right)=a,\left(1 \cdot a_{<-1>}\right) a_{<0>}=a$, and

$$
\begin{aligned}
& b \cdot\left(a_{(0)}\left(a_{(1)} \cdot a^{\prime}\right)\right)=a_{(0)_{R}}\left(b_{R} a_{(1)} \cdot a^{\prime}\right) \\
& \left(\left(a \cdot a_{<-1>}^{\prime}\right) a_{<0>}^{\prime}\right) \cdot b=\left(a \cdot a_{<-1>}^{\prime} b_{Q}\right) a_{<0>Q}^{\prime} \\
& \rho_{r}\left(a \bullet a^{\prime}\right)=\left(a_{(0)} \cdot a_{(0)_{R_{<-1>}}}^{\prime}\right) a_{(0)_{R_{<0>}}^{\prime}}^{\prime} \otimes a_{(1)_{R}} a_{(1)}^{\prime}, \\
& \rho_{l}\left(a \bullet a^{\prime}\right)=a_{<-1>} a_{<-1>_{Q}}^{\prime} \otimes a_{<0>Q_{(0)}}\left(a_{<0>Q_{(1)}} \cdot a_{<0>}^{\prime}\right) \\
& a_{(0)_{<-1>}} \otimes a_{(0)_{<0>}} \otimes a_{(1)}=a_{<-1>} \otimes a_{<0>(0)} \otimes a_{<0>(1)}, \\
& a_{Q_{(0)}} \otimes a_{Q_{(1)}} \otimes b_{Q}=a_{(0)_{Q}} \otimes a_{(1)} \otimes b_{Q}, \\
& a_{R_{<-1>}} \otimes a_{R_{<0>}} \otimes b_{R}=a_{<-1>} \otimes a_{<0>_{R}} \otimes b_{R},
\end{aligned}
$$

for all $a, a^{\prime} \in A$ and $b \in B$. Then $(A, \bullet, 1)$ is an associative unital algebra, denoted in what follows by $\tilde{A}$.

Proof. Obviously, 1 is the unit, so we only prove the associativity of $\bullet$; we compute:

$$
\begin{aligned}
\left(a \bullet a^{\prime}\right) \bullet a^{\prime \prime} & =\left(\left(a \bullet a^{\prime}\right)_{(0)} \cdot a_{<-1>}^{\prime \prime}\right)\left(\left(a \bullet a^{\prime}\right)_{(1)} \cdot a_{<0>}^{\prime \prime}\right) \\
& \stackrel{4.3}{=}\left[\left(\left(a_{(0)} \cdot a_{(0)_{R_{<-1>}}}^{\prime}\right) a_{(0)_{R_{<0>}}}^{\prime}\right) \cdot a_{<-1>}^{\prime \prime}\right]\left[a_{(1)_{R}} a_{(1)}^{\prime} \cdot a_{<0>}^{\prime \prime}\right] \\
& \stackrel{4.2}{=}\left(a_{(0)} \cdot a_{(0)_{R_{<-1>}}^{\prime}}^{\prime} a_{<-1>_{Q}}^{\prime \prime}\right) a_{(0)_{R_{<0>}}}^{\prime}\left(a_{(1)_{R}} a_{(1)}^{\prime} \cdot a_{<0>}^{\prime \prime}\right) \\
& \stackrel{4.7}{=}\left(a_{(0)} \cdot a_{(0)_{<-1>}}^{\prime} a_{<-1>_{Q}}^{\prime \prime}\right) a_{(0)_{<0 R_{Q}}}^{\prime}\left(a_{(1)_{R}} a_{(1)}^{\prime} \cdot a_{<0>}^{\prime \prime}\right)
\end{aligned}
$$




$$
\begin{aligned}
& \stackrel{4.5}{=}\left(a_{(0)} \cdot a_{<-1>}^{\prime} a_{<-1>_{Q}}^{\prime \prime}\right) a_{<0>_{(0)} R_{Q}}^{\prime}\left(a_{(1)_{R}} a_{<0>(1)}^{\prime} \cdot a_{<0>}^{\prime \prime}\right), \\
& a \bullet\left(a^{\prime} \bullet a^{\prime \prime}\right)=\left(a_{(0)} \cdot\left(a^{\prime} \bullet a^{\prime \prime}\right)_{<-1>}\right)\left(a_{(1)} \cdot\left(a^{\prime} \bullet a^{\prime \prime}\right)_{<0>}\right) \\
& \stackrel{4.4}{=}\left(a_{(0)} \cdot a_{<-1>}^{\prime} a_{<-1>Q}^{\prime \prime}\right)\left(a_{(1)} \cdot\left(a_{<0>Q_{(0)}}^{\prime}\left(a_{<0>Q_{(1)}}^{\prime} \cdot a_{<0>}^{\prime \prime}\right)\right)\right) \\
& \stackrel{4.1}{=}\left(a_{(0)} \cdot a_{<-1>}^{\prime} a_{<-1>Q}^{\prime \prime}\right) a_{<0>Q_{(0)}}^{\prime}\left(a_{(1)_{R}} a_{<0>Q_{(1)}}^{\prime} \cdot a_{<0>}^{\prime \prime}\right) \\
& \stackrel{\sqrt[4.6]{=}}{=}\left(a_{(0)} \cdot a_{<-1>}^{\prime} a_{<-1>Q}^{\prime \prime}\right) a_{<0>(0) Q_{R}}^{\prime}\left(a_{(1)_{R}} a_{<0>(1)}^{\prime} \cdot a_{<0>}^{\prime \prime}\right) \\
& \stackrel{2.7}{=}\left(a_{(0)} \cdot a_{<-1>}^{\prime} a_{<-1>_{Q}}^{\prime \prime}\right) a_{<0>_{(0)} R_{Q}}^{\prime}\left(a_{(1)_{R}} a_{<0>(1)}^{\prime} \cdot a_{<0>}^{\prime \prime}\right) \text {, }
\end{aligned}
$$

finishing the proof.

Theorem 4.3 Assume that the hypotheses of Proposition 4.2 are satisfied, such that moreover $A \otimes_{Q} B$ is an L-R-twisted tensor product of algebras. Assume also that we are given linear maps $\lambda_{r}: A \rightarrow A \otimes B, \lambda_{r}(a)=a_{[0]} \otimes a_{[1]}$, and $\lambda_{l}: A \rightarrow B \otimes A, \lambda_{l}(a)=a_{\{-1\}} \otimes a_{\{0\}}$, such that $\lambda_{r}(1)=1 \otimes 1, \lambda_{l}(1)=1 \otimes 1$ and the following relations hold:

$$
\begin{aligned}
& a_{(0)_{[0]}} \otimes a_{(0)_{[1]}} a_{(1)}=a \otimes 1, \\
& a_{[0]_{(0)}} \otimes a_{[0]_{(1)}} a_{[1]}=a \otimes 1 \text {, } \\
& a_{<-1>} a_{<0>_{\{-1\}}} \otimes a_{<0>\{0\}}=1 \otimes a, \\
& a_{\{-1\}} a_{\{0\}<-1>} \otimes a_{\{0\}<0>}=1 \otimes a, \\
& a_{[0]_{\{-1\}}} \otimes a_{[0]_{\{0\}}} \otimes a_{[1]}=a_{\{-1\}} \otimes a_{\{0\}_{[0]}} \otimes a_{\{0\}_{[1]}}, \\
& a_{[0]_{<-1>}} \otimes a_{[0]_{<0>}} \otimes a_{[1]}=a_{<-1>} \otimes a_{<0>[0]} \otimes a_{<0>[1]}, \\
& a_{(0)_{\{-1\}}} \otimes a_{(0)_{\{0\}}} \otimes a_{(1)}=a_{\{-1\}} \otimes a_{\{0\}_{(0)}} \otimes a_{\{0\}_{(1)}}, \\
& \lambda_{r}\left(a a^{\prime}\right)=a_{[0]_{(0)}}\left(a_{[0]_{(1)}} \cdot a_{R_{[0]}}^{\prime}\right) \otimes a_{R_{[1]}}^{\prime} a_{[1]_{R}}, \\
& \lambda_{l}\left(a a^{\prime}\right)=a_{\{-1\}_{Q}}^{\prime} a_{Q_{\{-1\}}} \otimes\left(a_{Q_{\{0\}}} \cdot a_{\{0\}<-1>}^{\prime}\right) a_{\{0\}<0>}^{\prime}, \\
& \rho_{l}\left(\left(a \cdot a_{<-1>}^{\prime}\right) a_{<0>}^{\prime}\right)=a_{<-1>} a_{<-1>_{Q}}^{\prime} \otimes a_{<0>Q} a_{<0>}^{\prime}, \\
& a_{(0)_{<-1>R}} a_{(1)_{Q}}^{\prime} \otimes a_{(0)_{<0>}} \otimes a_{(0)_{<0>_{R}}^{\prime}}^{\prime} \otimes a_{(0)_{<-1>}^{\prime}}^{\prime} \otimes a_{(1)} \\
& =a_{(1)}^{\prime} a_{(0)_{<-1>}} \otimes a_{(0)_{<0>}} \otimes a_{(0)_{<0>}}^{\prime} \otimes a_{(0)_{<-1>}}^{\prime} \otimes a_{(1)}, \\
& a_{<0>Q_{\{-1\}_{R}}^{\prime}}^{\prime} a_{(1)} \otimes a_{<0>Q_{\{0\}}}^{\prime} \otimes a_{<-1>}^{\prime} \otimes a_{(0)_{R}} \otimes b_{Q} \\
& =a_{(1)_{q}} a_{<0>Q_{q_{\{-1\}}}^{\prime}}^{\prime} \otimes a_{<0>_{Q_{\{0\}}}^{\prime}}^{\prime} \otimes a_{<-1>}^{\prime} \otimes a_{(0)} \otimes b_{Q}, \\
& a_{(0)_{R_{[0]}}} \otimes a_{<-1>}^{\prime} a_{(0)_{R_{[1]_{Q}}}} \otimes a_{<0>Q}^{\prime} \otimes a_{(1)} \otimes b_{R} \\
& =a_{(0)_{r_{R}}} \otimes a_{(0)_{r_{R_{[1]}}}} a_{<-1>R}^{\prime} \otimes a_{<0>}^{\prime} \otimes a_{(1)} \otimes b_{r}, \\
& a_{<0>Q_{\{0\}_{(0)}}} \otimes a_{<0>Q_{\{0\}_{(1)}}} \otimes a_{<-1>} \otimes a_{<0>_{Q_{\{-1\}}}} \otimes b_{R} \otimes b_{Q}^{\prime}
\end{aligned}
$$

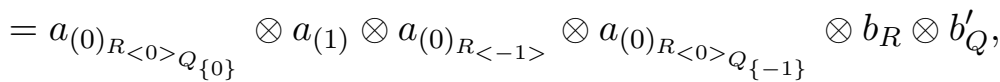

$$
\begin{aligned}
& a_{(0)_{R_{[1]}}} \otimes a_{(0)_{R_{[0]}<0>Q}} \otimes a_{(0)_{R_{[0]_{<-1>}}}} \otimes a_{(1)} \otimes b_{R} \otimes b_{Q}^{\prime}
\end{aligned}
$$




$$
\begin{gathered}
=a_{(0)_{<0>} R_{Q_{[1]}}} \otimes a_{(0)_{<0>} R_{Q_{[0]}}} \otimes a_{(0)_{<-1>}} \otimes a_{(1)} \otimes b_{R} \otimes b_{Q}^{\prime}, \\
\left(a_{(0)} \cdot b^{\prime}\right)_{R_{[0]}(0)} \otimes\left(a_{(0)} \cdot b^{\prime}\right)_{R_{[0]_{(1)}}} \otimes\left(a_{(0)} \cdot b^{\prime}\right)_{R_{[1]}} \otimes b_{R} \otimes a_{(1)} \\
=a_{(0)_{R_{[0]}}(0)} \cdot b^{\prime} \otimes a_{(0)_{R_{[0]}}} \otimes a_{(0)_{R_{[1]}}} \otimes b_{R} \otimes a_{(1)}, \\
a_{<-1>}^{\prime} \otimes\left(b \cdot a_{<0>}^{\prime}\right)_{Q_{\{-1\}}} \otimes\left(b \cdot a_{<0>}^{\prime}\right)_{Q_{\{0\}<-1>}} \otimes\left(b \cdot a_{<0>}^{\prime}\right)_{Q_{\{0\}<0>}} \otimes b_{Q}^{\prime} \\
=a_{<-1>}^{\prime} \otimes a_{<0>Q_{\{-1\}}}^{\prime} \otimes a_{<0>Q_{\{0\}<-1>}}^{\prime} \otimes b \cdot a_{<0>_{Q_{\{0\}}}^{\prime}} \otimes b_{Q}^{\prime},
\end{gathered}
$$

for all $a, a^{\prime} \in A$ and $b, b^{\prime} \in B$. Define the maps

$$
\begin{aligned}
& \tilde{R}: B \otimes \tilde{A} \rightarrow \tilde{A} \otimes B, \quad \tilde{R}(b \otimes a)=a_{(0)_{R_{[0]}}} \otimes a_{(0)_{R_{[1]}}} b_{R} a_{(1)}, \\
& \tilde{Q}: \tilde{A} \otimes B \rightarrow \tilde{A} \otimes B, \quad \tilde{Q}(a \otimes b)=a_{<0>_{Q_{\{0\}}}} \otimes a_{<-1>} b_{Q} a_{<0>Q_{\{-1\}}} .
\end{aligned}
$$

Then $\tilde{A}_{\tilde{Q}} \otimes_{\tilde{R}} B$ is an L-R-twisted tensor product of algebras and we have an algebra isomorphism

$$
\tilde{A}_{\tilde{Q}} \otimes_{\tilde{R}} B \simeq A_{Q} \otimes_{R} B, \quad a \otimes b \mapsto a_{(0)_{<0>}} \otimes a_{(1)} b a_{(0)_{<-1>}} .
$$

Proof. We have to prove that $\tilde{R}$ and $\tilde{Q}$ satisfy (2.1) - (2.8) for the algebras $\tilde{A}$ and $B$. We will only prove (2.2) $,(2.5),(2.7),(2.8)$, while (2.1) $,(2.4),(2.3),(2.6)$ are much easier and are left to the reader.

Proof of (2.2)

$$
\begin{aligned}
& \left(a \bullet a^{\prime}\right)_{\tilde{R}} \otimes b_{\tilde{R}} \quad=\quad\left(a \bullet a^{\prime}\right)_{(0)_{R_{[0]}}} \otimes\left(a \bullet a^{\prime}\right)_{(0)_{R_{[1]}}} b_{R}\left(a \bullet a^{\prime}\right)_{(1)} \\
& \stackrel{4.3}{=}\left[\left(a_{(0)} \cdot a_{(0)_{r_{<-1>}}^{\prime}}^{\prime}\right) a_{(0)_{r_{<0>}}^{\prime}}\right]_{R_{[0]}} \\
& \otimes\left[\left(a_{(0)} \cdot a_{(0)_{r<-1>}}^{\prime}\right) a_{(0)_{r_{<0>}}}^{\prime}\right]_{R_{[1]}} b_{R} a_{(1)_{r}} a_{(1)}^{\prime} \\
& \stackrel{2.2}{=}\left[\left(a_{(0)} \cdot a_{(0)_{r<-1>}}^{\prime}\right)_{R} a_{(0)_{r_{<0>\mathcal{R}}}}^{\prime}\right]_{[0]} \\
& \otimes\left[\left(a_{(0)} \cdot a_{(0)_{r_{<-1>}}}^{\prime}\right)_{R} a_{(0)_{r_{<0>\mathcal{R}}}}^{\prime}\right]_{[1]} b_{R_{\mathcal{R}}} a_{(1)_{r}} a_{(1)}^{\prime} \\
& \stackrel{4.7}{=}\left[\left(a_{(0)} \cdot a_{(0)_{<-1>}}^{\prime}\right)_{R} a_{(0)_{<0>r_{\mathcal{R}}}}\right]_{[0]} \\
& \otimes\left[\left(a_{(0)} \cdot a_{(0)_{<-1>}}^{\prime}\right)_{R} a_{(0)_{<0>r_{\mathcal{R}}}^{\prime}}^{\prime}\right]_{[1]} b_{R_{\mathcal{R}}} a_{(1)_{r}} a_{(1)}^{\prime}
\end{aligned}
$$

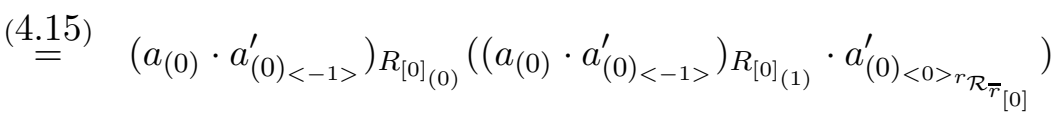

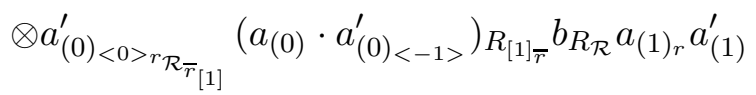

$$
\begin{aligned}
& \stackrel{4.23}{=}\left(a_{(0)_{R_{[0]}(0)}} \cdot a_{(0)_{<-1>}}^{\prime}\right)\left(a_{(0)_{R_{[0]}(1)}} \cdot a_{(0)_{<0>r_{\mathcal{R}_{\bar{r}}}[0]}^{\prime}}\right)
\end{aligned}
$$

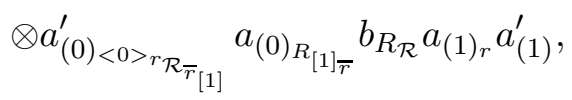

$$
\begin{aligned}
& a_{\tilde{R}} \bullet a_{\tilde{r}}^{\prime} \otimes b_{\tilde{R}_{\tilde{r}}} \quad=\quad a_{(0)_{R_{[0]}}} \bullet a_{\tilde{r}}^{\prime} \otimes\left(a_{(0)_{R_{[1]}}} b_{R} a_{(1)}\right)_{\tilde{r}} \\
& =\quad a_{(0)_{R_{[0]}}} \bullet a_{(0)_{[0]}}^{\prime} \otimes a_{(0)_{r_{[1]}}}^{\prime}\left(a_{(0)_{R_{[1]}}} b_{R} a_{(1)}\right)_{r} a_{(1)}^{\prime}
\end{aligned}
$$




$$
\begin{aligned}
& \stackrel{[2.31}{=} \quad a_{(0)_{R_{[0]}}} \bullet a_{(0)_{r_{\mathcal{R}}[0]}^{\prime}}^{\prime} \otimes a_{(0)_{r_{\mathcal{R}}[1]}}^{\prime} a_{(0)_{R_{[1]}}} b_{R_{\mathcal{R}}} a_{(1)_{r}} a_{(1)}^{\prime} \\
& =\left(a_{(0)_{R_{[0]}}(0)} \cdot a_{(0)_{r_{\mathcal{R}}[0]_{<-1>}}^{\prime}}\right)\left(a_{(0)_{R_{[0]}}} \cdot a_{(0)_{r_{\mathcal{R}}[0]_{<0>}}}^{\prime}\right) \\
& \otimes a_{(0)_{r_{\mathcal{R}[1]}}}^{\prime} a_{(0)_{R_{[1]}}} b_{R_{\mathcal{R}}} a_{(1)_{r}} a_{(1)}^{\prime} \\
& \stackrel{4.13}{=}\left(a_{(0)_{R_{[0]}}(0)} \cdot a_{(0)_{r_{\mathcal{R}<-1>}}^{\prime}}^{\prime}\right)\left(a_{(0)_{R_{[0]}(1)}} \cdot a_{(0)_{r_{\mathcal{R}<0>}[0]}}^{\prime}\right) \\
& \otimes a_{(0)_{r_{\mathcal{R}}<0>}[1]}^{\prime} a_{(0)_{R_{[1] \bar{r}}}} b_{R_{\mathcal{R}}} a_{(1)_{r}} a_{(1)}^{\prime}
\end{aligned}
$$

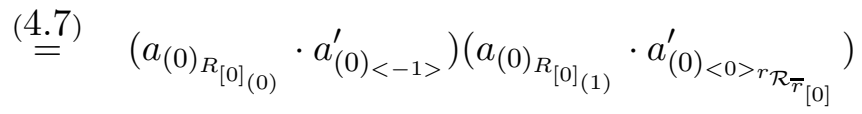

$$
\begin{aligned}
& \otimes a_{(0)_{<0>r_{\mathcal{R}_{\bar{r}}[1]}}^{\prime}}^{\prime} a_{(0)_{R_{[1] \bar{r}}}} b_{R_{\mathcal{R}}} a_{(1)_{r}} a_{(1)}^{\prime} \text {, q.e.d. }
\end{aligned}
$$

Proof of (2.5)

$$
\begin{aligned}
& \left(a \bullet a^{\prime}\right)_{\tilde{Q}} \otimes b_{\tilde{Q}} \quad=\quad\left(a \bullet a^{\prime}\right)_{<0>Q_{\{0\}}} \otimes\left(a \bullet a^{\prime}\right)_{<-1>} b_{Q}\left(a \bullet a^{\prime}\right)_{<0>Q_{\{-1\}}} \\
& \stackrel{4.47, \text {, (4.6) }}{=}\left[a_{<0>(0)_{q}}\left(a_{<0>(1)} \cdot a_{<0>}^{\prime}\right)\right]_{Q_{\{0\}}} \otimes a_{<-1>} a_{<-1>_{q}}^{\prime} b_{Q} \\
& {\left[a_{<0>(0) q}\left(a_{<0>(1)} \cdot a_{<0>}^{\prime}\right)\right]_{Q_{\{-1\}}}} \\
& \stackrel{2.5}{=}\left[a_{<0>{ }_{(0) q_{\bar{q}}}}\left(a_{<0>(1)} \cdot a_{<0>}^{\prime}\right)_{Q}\right]_{\{0\}} \otimes a_{<-1>} a_{<-1>}^{\prime} b_{Q_{\bar{q}}} \\
& {\left[a_{<0>(0)_{\bar{q}}}\left(a_{<0>(1)} \cdot a_{<0>}^{\prime}\right)_{Q}\right]_{\{-1\}}} \\
& \stackrel{4.16}{=}\left(a_{<0>(0) q_{\bar{q}} \bar{Q}_{\{0\}}} \cdot\left(a_{<0>(1)} \cdot a_{<0>}^{\prime}\right)_{Q_{\{0\}}}\right)\left(a_{<0>(1)} \cdot a_{<0>}^{\prime}\right)_{Q_{\{0\}}<0>} \\
& a_{<-1>} a_{<-1>q}^{\prime} b_{Q_{\bar{q}}}\left(a_{<0>(1)} \cdot a_{<0>}^{\prime}\right)_{Q_{\{-1\} \bar{Q}}} a_{<0>(0) q_{\bar{q}} \bar{Q}_{\{-1\}}} \\
& \stackrel{4.24}{=}\left(a_{<0>(0) q_{\bar{q}} \bar{Q}_{\{0\}}} \cdot a_{<0>Q_{\{0\}}}^{\prime}\right)\left(a_{<0>}{ }_{(1)} \cdot a_{<0>Q_{\{0\}<0>}}^{\prime}\right) \\
& a_{<-1>} a_{<-1>q}^{\prime} b_{Q_{\bar{q}}} a_{<0>Q_{\{-1\}}}^{\prime} a_{<0>{ }_{(0)} q_{\bar{q}} \bar{Q}_{\{-1\}}}, \\
& a_{\tilde{q}} \bullet a_{\tilde{Q}}^{\prime} \otimes b_{\tilde{Q}_{\tilde{q}}} \quad=\quad a_{\tilde{q}} \bullet a_{<0>Q_{\{0\}}}^{\prime} \otimes\left(a_{<-1>}^{\prime} b_{Q} a_{<0>Q_{\{-1\}}}^{\prime}\right)_{\tilde{q}} \\
& =\quad a_{<0>_{\{0\}}} \bullet a_{<0>Q_{\{0\}}}^{\prime} \otimes a_{<-1>}\left(a_{<-1>}^{\prime} b_{Q} a_{<0>_{Q_{\{-1\}}}^{\prime}}\right)_{q} a_{<0>_{q_{\{-1\}}}} \\
& \stackrel{\text { 2.6. }}{=} a_{<0>{ }_{q_{\bar{q}} \bar{Q}_{\{0\}}}} \bullet a_{<0>Q_{\{0\}}}^{\prime} \otimes a_{<-1>} a_{<-1>q}^{\prime} b_{Q_{\bar{q}}} a_{<0>Q_{\{-1\}}}^{\prime} a_{<0>{ }_{q_{\bar{q}}}} \\
& =\left(a_{<0>{ }_{q_{\bar{q}}} \bar{Q}_{\{0\}}(0)} \cdot a_{<0>Q_{\{0\}}}^{\prime}\right)\left(a_{<0>-1>}{ }_{q_{\bar{q}} \bar{Q}_{\{0\}}} \cdot a_{<0>Q_{\{0\}}}^{\prime}\right) \\
& \otimes a_{<-1>} a_{<-1>q}^{\prime} b_{Q_{\bar{q}}} a_{<0>Q_{\{-1\}}}^{\prime} a_{<0>_{q_{\bar{q}}}} \\
& \stackrel{\text { 4.14 }}{=}\left(a_{<0>{ }_{q_{\bar{q}} \bar{Q}_{(0)}\{0\}}} \cdot a_{<0>Q_{\{0\}}}^{\prime}\right)\left(a_{<0>>_{q_{\bar{q}} \bar{Q}_{(1)}}} \cdot a_{<0>Q_{\{0\}<0>}}^{\prime}\right)
\end{aligned}
$$




$$
\begin{aligned}
& \otimes a_{<-1>} a_{<-1>q}^{\prime} b_{Q_{\bar{q}}} a_{<0>Q_{\{-1\}}}^{\prime} a_{<0>{ }_{q_{\bar{Q}}}}{ }_{{ }_{(0)}\{-1\}} \\
& \stackrel{[4.6]}{=}\left(a_{<0>{ }_{(0)}{ }_{{ }_{\bar{q}}} \bar{Q}_{\{0\}}} \cdot a_{<0>Q_{\{0\}<-1>}}^{\prime}\right)\left(a_{<0>(1)} \cdot a_{<0>}^{\prime}{ }_{Q_{\{0\}<0>}}\right) \\
& a_{<-1>} a_{<-1>q}^{\prime} b_{Q_{\bar{q}}} a_{<0>Q_{\{-1\}}}^{\prime} a_{<0>(0)_{q_{\bar{q}}} \bar{Q}_{\{-1\}}}, \quad \text { q.e.d. }
\end{aligned}
$$

Proof of (2.7)

$$
\begin{aligned}
& b_{\tilde{R}} \otimes a_{\tilde{R}_{\tilde{Q}}} \otimes b_{\tilde{Q}}^{\prime}=a_{(0)_{R_{[1]}}} b_{R} a_{(1)} \otimes\left(a_{(0)_{R_{[0]}}}\right)_{\tilde{Q}} \otimes b_{\tilde{Q}}^{\prime} \\
& =\quad a_{(0)_{R_{[1]}}} b_{R} a_{(1)} \otimes a_{(0)_{\left.R_{[0]}\right)>Q_{\{0\}}}} \otimes a_{(0)_{R_{[0]<-1>}}} b_{Q}^{\prime} a_{(0)_{R_{[0]}<0>} Q_{\{-1\}}} \\
& \stackrel{4.22}{=} a_{(0)_{<0>} R_{Q_{[1]}}} b_{R} a_{(1)} \otimes a_{(0)_{<0>} R_{Q_{[0]}}} \otimes a_{(0)<-1>} b_{Q}^{\prime} a_{(0)_{<0>} R_{Q_{[0]}}} \\
& \stackrel{4.12}{=} a_{(0)_{<0>} R_{Q_{\{0\}}[1]}} b_{R} a_{(1)} \otimes a_{(0)_{<0>} R_{Q_{\{0\}}}} \otimes a_{(0)<-1>} b_{Q}^{\prime} a_{(0)_{<0>} R_{Q_{\{-1\}}}}, \\
& \begin{aligned}
b_{\tilde{R}} \otimes a_{\tilde{Q}_{\tilde{R}}} \otimes b_{\tilde{Q}}^{\prime} & =b_{\tilde{R}} \otimes\left(a_{<0>Q_{\{0\}}}\right)_{\tilde{R}} \otimes a_{<-1>} b_{Q}^{\prime} a_{<0>Q_{\{-1\}}} \\
& =a_{<0>Q_{\{0\}}(0)_{R_{[1]}}} b_{R} a_{<0>Q_{\{0\}}(1)} \otimes a_{<0>Q_{\{0\}}(0)_{R_{[0]}}} \otimes a_{<-1>} b_{Q}^{\prime} a_{<0>Q_{\{-1\}}}
\end{aligned} \\
& \stackrel{\text { 4.21 }}{=} a_{(0)_{R_{<0>} Q_{\{0\}}[1]}} b_{R} a_{(1)} \otimes a_{(0)_{R_{<0>}} Q_{\{0\}}[0]} \otimes a_{(0)_{R_{<-1>}}} b_{Q}^{\prime} a_{(0)_{R_{<0>} Q_{\{-1\}}}} \\
& \stackrel{\text { 4.77 }}{=} a_{(0)_{<0>} R_{Q_{\{0\}}[1]}} b_{R} a_{(1)} \otimes a_{(0)_{<0>}{ }_{Q_{Q_{\{0\}}}}} \otimes a_{(0)<-1>} b_{Q}^{\prime} a_{(0)_{<0>} R_{Q_{\{-1\}}}} \text {, q.e.d. }
\end{aligned}
$$

Proof of (2.8)

$$
\begin{aligned}
& a_{\tilde{R}} \otimes b_{\tilde{R}_{\tilde{Q}}} \otimes a_{\tilde{Q}}^{\prime}=a_{(0)_{r[0]}} \otimes\left(a_{(0)_{r[1]}} b_{r} a_{(1)}\right)_{\tilde{Q}} \otimes a_{\tilde{Q}}^{\prime} \\
& =\quad a_{(0)_{r[0]}} \otimes a_{<-1>}^{\prime}\left(a_{(0)_{[1]}} b_{r} a_{(1)}\right)_{Q} a_{<0>Q_{\{-1\}}}^{\prime} \otimes a_{<0>Q_{\{0\}}}^{\prime}
\end{aligned}
$$

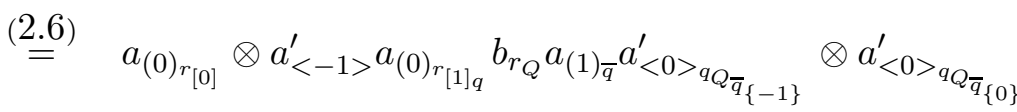

$$
\begin{aligned}
& \stackrel{4.20}{=} a_{\left.(0)_{r_{R}}\right]} \otimes a_{(0)_{r_{R}}} a_{<-1>{ }_{R}}^{\prime} b_{r_{Q}} a_{(1)_{q}} a_{<0>Q_{q_{\{-1\}}}^{\prime}}^{\prime} \otimes a_{<0>Q_{q_{\{0\}}}}^{\prime}, \\
& a_{\tilde{R}} \otimes b_{\tilde{Q}_{\tilde{R}}} \otimes a_{\tilde{Q}}^{\prime}=a_{\tilde{R}} \otimes\left(a_{<-1>}^{\prime} b_{Q} a_{<0>Q_{\{-1\}}}^{\prime}\right)_{\tilde{R}} \otimes a_{<0>Q_{\{0\}}}^{\prime} \\
& =\quad a_{(0)_{R_{[0]}}} \otimes a_{(0)_{R_{[1]}}}\left(a_{<-1>}^{\prime} b_{Q} a_{<0>_{Q_{\{-1\}}}^{\prime}}\right)_{R} a_{(1)} \otimes a_{<0>Q_{\{0\}}}^{\prime} \\
& \stackrel{2.3}{=} a_{(0)_{R_{r}}[0]} \otimes a_{(0)_{R_{r_{\mathcal{R}}}}} a_{<-1>\mathcal{R}}^{\prime} b_{Q_{r}} a_{<0>Q_{\{-1\}}}^{\prime} a_{(1)} \otimes a_{<0>Q_{\{0\}}}^{\prime}
\end{aligned}
$$

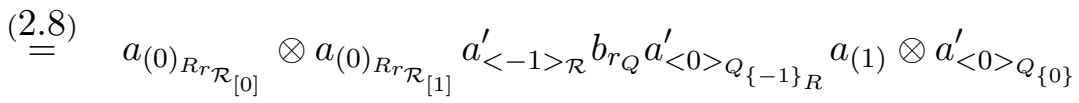




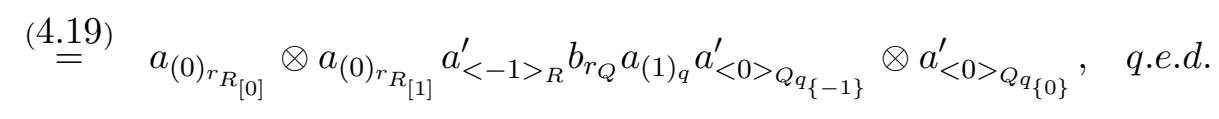

We prove now that the map $\varphi: \tilde{A}_{\tilde{Q}} \otimes_{\tilde{R}} B \rightarrow A_{Q} \otimes_{R} B, \varphi(a \otimes b)=a_{(0)_{<0>}} \otimes a_{(1)} b a_{(0)_{<-1>}>}$, is an algebra isomorphism. First, using (4.5), (4.8), (4.10), (4.12), (4.9), (4.11), it is easy to see that $\varphi$ is bijective, with inverse given by $a \otimes b \mapsto a_{[0]_{\{0\}}} \otimes a_{[1]} b a_{[0]_{\{-1\}}}$. It is obvious that $\varphi(1 \otimes 1)=1 \otimes 1$, so we only have to prove that $\varphi$ is multiplicative. We compute:

$$
\begin{aligned}
& \varphi\left((a \otimes b)\left(a^{\prime} \otimes b^{\prime}\right)\right)=\varphi\left(a_{\tilde{Q}} \bullet a_{\tilde{R}}^{\prime} \otimes b_{\tilde{R}} b_{\tilde{Q}}^{\prime}\right) \\
& =\varphi\left(a_{<0>Q_{\{0\}}} \bullet a_{(0)_{R_{[0]}}}^{\prime} \otimes a_{(0)_{R_{[1]}}}^{\prime} b_{R} a_{(1)}^{\prime} a_{<-1>} b_{Q}^{\prime} a_{<0>Q_{\{-1\}}}\right) \\
& =\left[a_{<0>Q_{\{0\}}} \bullet a_{(0)_{R_{[0]}}}^{\prime}\right]_{(0)_{<0>}} \otimes\left[a_{<0>Q_{\{0\}}} \bullet a_{(0)_{R_{[0]}}}^{\prime}\right]_{(1)} a_{(0)_{R_{[1]}}}^{\prime} b_{R} a_{(1)}^{\prime} \\
& a_{<-1>} b_{Q}^{\prime} a_{<0>Q_{\{-1\}}}\left[a_{<0>Q_{\{0\}}} \bullet a_{(0)_{R_{[0]}}}^{\prime}\right]_{(0)_{<-1>}} \\
& \stackrel{4.3}{=}\left[\left(a_{<0>_{Q_{\{0\}}(0)}} \cdot a_{(0)_{R_{[0]}(0)_{r}<-1>}}^{\prime}\right) a_{(0)_{R_{[0]}(0)_{r}<0>}}^{\prime}\right]_{<0>} \\
& \otimes a_{<0>Q_{\{0\}}(1)_{r}} a_{(0)_{R_{[0]}(1)}^{\prime}}^{\prime} a_{(0)_{R_{[1]}}}^{\prime} b_{R} a_{(1)}^{\prime} a_{<-1>} b_{Q}^{\prime} a_{<0>Q_{\{-1\}}} \\
& {\left[\left(a_{<0>_{Q_{\{0\}}(0)}} \cdot a_{(0)_{R_{[0]}(0)_{r}<-1>}^{\prime}}^{\prime}\right) a_{(0)_{R_{[0]}(0)_{r}<0>}^{\prime}}\right]_{<-1>}} \\
& \stackrel{4.9}{=}\left[\left(a_{<0>Q_{\{0\}}(0)} \cdot a_{(0)_{R_{r}<-1>}^{\prime}}^{\prime}\right) a_{(0)_{R_{r}<0>}^{\prime}}^{\prime}\right]_{<0>} \\
& \otimes a_{<0>Q_{\{0\}}(1) r} b_{R} a_{(1)}^{\prime} a_{<-1>} b_{Q}^{\prime} a_{<0>Q_{\{-1\}}} \\
& {\left[\left(a_{<0>_{Q_{\{0\}}(0)}} \cdot a_{(0)_{R_{r}<-1>}}^{\prime}\right) a_{(0)_{R_{r}<0>}}^{\prime}\right]_{<-1>}} \\
& \stackrel{4.17}{=} \\
& a_{<0>Q_{\{0\}}(0)_{<0>q}} a_{(0)_{R_{r}<0>}}^{\prime} \otimes a_{<0>Q_{\{0\}_{(1) r}}} b_{R} a_{(1)}^{\prime} a_{<-1>} b_{Q}^{\prime} \\
& a_{<0>Q_{\{-1\}}} a_{<0>Q_{\{0\}}(0)_{<-1>}} a_{(0)_{R_{r}<-1>q}}^{\prime} \\
& 4.5 \\
& a_{<0>Q_{\{0\}<0>(0)_{q}}} a_{(0)_{R_{r}<0>}^{\prime}}^{\prime} \otimes a_{<0>Q_{\{0\}<0>(1)_{r}}} b_{R} a_{(1)}^{\prime} a_{<-1>} b_{Q}^{\prime} \\
& a_{<0>Q_{\{-1\}}} a_{<0>Q_{\{0\}<-1>}} a_{(0)_{R_{r}<-1>q}}^{\prime} \\
& 4.11 \\
& a_{<0>Q_{(0) q}} a_{(0)_{R_{r}<0>}}^{\prime} \otimes a_{<0>Q_{(1) r}} b_{R} a_{(1)}^{\prime} a_{<-1>} b_{Q}^{\prime} a_{(0)_{R_{r}<-1>q}}^{\prime} \\
& \stackrel{\text { 4.6. }}{=} a_{<0>(0) Q_{Q q}} a_{(0)_{R_{r}<0>}^{\prime}}^{\prime} \otimes a_{<0>(1)_{r}} b_{R} a_{(1)}^{\prime} a_{<-1>} b_{Q}^{\prime} a_{(0)_{R_{r}<-1>_{q}}^{\prime}} \\
& \stackrel{4.5}{=} a_{(0)_{<0>Q_{q}}} a_{(0)_{R_{r}<0>}}^{\prime} \otimes a_{(1)_{r}} b_{R} a_{(1)}^{\prime} a_{(0)_{<-1>}} b_{Q}^{\prime} a_{(0)_{R_{r}<-1>_{q}}}^{\prime} \\
& \stackrel{4.7}{=} a_{(0)_{<0>Q_{q}}} a_{(0)_{<0\rangle_{r}}^{\prime}}^{\prime} \otimes a_{(1)_{r}} b_{R} a_{(1)}^{\prime} a_{(0)<-1>} b_{Q}^{\prime} a_{(0)_{<-1>q}}^{\prime} \text {, } \\
& \varphi(a \otimes b) \varphi\left(a^{\prime} \otimes b^{\prime}\right) \quad=\quad\left(a_{(0)_{<0>}} \otimes a_{(1)} b a_{\left.(0)_{<-1>}\right)}\right)\left(a_{(0)_{<0>}}^{\prime} \otimes a_{(1)}^{\prime} b^{\prime} a_{(0)_{<-1>}}^{\prime}\right) \\
& =\quad a_{(0)_{<0>Q}} a_{(0)_{<0>R}}^{\prime} \otimes\left(a_{(1)} b a_{\left.(0)_{<-1>}\right)}\right)_{R}\left(a_{(1)}^{\prime} b^{\prime} a_{(0)_{<-1>}}^{\prime}\right)_{Q} \\
& \stackrel{2.3], 2.6}{=} a_{(0)_{<0>Q_{q_{\bar{q}}}}} a_{(0)_{<0>_{R_{\bar{r}}}}^{\prime}} \otimes a_{(1)_{\bar{r}}} b_{r} a_{(0)_{<-1>_{R}}} a_{(1)_{Q}}^{\prime} b_{q}^{\prime} a_{(0)_{<-1>_{\bar{q}}}}^{\prime}
\end{aligned}
$$




$$
\stackrel{4.18}{=} a_{(0)_{<0>Q_{q}}} a_{(0)_{<0>_{R}}}^{\prime} \otimes a_{(1)_{r}} b_{R} a_{(1)}^{\prime} a_{(0)_{<-1>}} b_{Q}^{\prime} a_{(0)_{<-1>q}}^{\prime}
$$

finishing the proof.

Remark 4.4 It is very easy to see that Theorem 4.3 generalizes Theorem 4.4 in [11]. On the other hand, it generalizes also Proposition 4.1. Indeed, Proposition 4.1 may be obtained by taking in Theorem 4.3 $A=\mathcal{A}, B=H, R: H \otimes \mathcal{A} \rightarrow \mathcal{A} \otimes H, R(h \otimes \varphi)=h_{1} \cdot \varphi \otimes h_{2}, Q: \mathcal{A} \otimes H \rightarrow \mathcal{A} \otimes H$, $Q(\varphi \otimes h)=\varphi \cdot h_{2} \otimes h_{1}, \mu_{l}: H \otimes \mathcal{A} \rightarrow \mathcal{A}, \mu_{l}(h \otimes \varphi)=h \cdot \varphi, \mu_{r}: \mathcal{A} \otimes H \rightarrow \mathcal{A}, \mu_{r}(\varphi \otimes h)=\varphi \cdot h$, $\rho_{r}: \mathcal{A} \rightarrow \mathcal{A} \otimes H, \rho_{r}(\varphi)=G^{1} \cdot \varphi \otimes G^{2}, \rho_{l}: \mathcal{A} \rightarrow H \otimes \mathcal{A}, \rho_{l}(\varphi)=F^{1} \otimes \varphi \cdot F^{2}, \lambda_{r}: \mathcal{A} \rightarrow \mathcal{A} \otimes H$, $\lambda_{r}(\varphi)=F^{1} \cdot \varphi \otimes F^{2}, \lambda_{l}: \mathcal{A} \rightarrow H \otimes \mathcal{A}, \lambda_{l}(\varphi)=G^{1} \otimes \varphi \cdot G^{2}$, for all $h \in H$ and $\varphi \in \mathcal{A}$.

\section{References}

[1] P. Bieliavsky, P. Bonneau, Y. Maeda, Universal deformation formulae, symplectic Lie groups and symmetric spaces, Pacific J. Math. 230 (2007), 41-57.

[2] P. Bieliavsky, P. Bonneau, Y. Maeda, Universal deformation formulae for three-dimensional solvable Lie groups, in "Quantum field theory and noncommutative geometry", 127-141, Lecture Notes in Phys., 662, Springer, Berlin, 2005.

[3] P. Bonneau, M. Gerstenhaber, A. Giaquinto, D, Sternheimer, Quantum groups and deformation quantization: Explicit approaches and implicit aspects, J. Math. Phys. 45 (2004), $3703-3741$.

[4] P. Bonneau, D. Sternheimer, Topological Hopf algebras, quantum groups and deformation quantization, in "Hopf algebras in noncommutative geometry and physics", 55-70, Lecture Notes in Pure and Appl. Math. 239, Marcel Dekker, New York, 2005.

[5] A. Borowiec, W. Marcinek, On crossed product of algebras, J. Math. Phys. 41 (2000), 6959-6975.

[6] D. Bulacu, F. Panaite, F. Van Oystaeyen, Quasi-Hopf algebra actions and smash products, Comm. Algebra 28 (2000), 631-651.

[7] D. Bulacu, F. Panaite, F. Van Oystaeyen, Generalized diagonal crossed products and smash products for quasi-Hopf algebras. Applications, Comm. Math. Phys. 266 (2006), 355-399.

[8] A. Cap, H. Schichl, J. Vanzura, On twisted tensor products of algebras, Comm. Algebra 23 (1995), 4701-4735.

[9] C. Cibils, Non-commutative duplicates of finite sets, J. Algebra Appl. 5 (2006), 361-377.

[10] F. Hausser, F. Nill, Diagonal crossed products by duals of quasi-quantum groups, Rev. Math. Phys. 11 (1999), 553-629.

[11] P. Jara Martínez, J. López Peña, F. Panaite, F. Van Oystaeyen, On iterated twisted tensor products of algebras, Internat. J. Math. 19 (2008), 1053-1101.

[12] P. Jara Martínez, J. López Peña, G. Navarro, D. Ştefan, On the classification of twisting maps between $K^{n}$ and $K^{m}$, to appear in Algebr. Represent. Theory 
[13] J. López Peña, F. Panaite, F. Van Oystaeyen, General twisting of algebras, Adv. Math. 212 (2007), 315-337.

[14] S. Majid, Quasi-* structure on q-Poincaré algebras, J. Geom. Phys. 22 (1997), 14-58.

[15] F. Panaite, F. Van Oystaeyen, L-R-smash product for (quasi-) Hopf algebras, J. Algebra 309 (2007), 168-191.

[16] F. Panaite, F. Van Oystaeyen, L-R-smash biproducts, double biproducts and a braided category of Yetter-Drinfeld-Long bimodules, arXiv:math.QA/0805.3432, to appear in Rocky Mount. J. Math.

[17] A. Van Daele, S. Van Keer, The Yang-Baxter and Pentagon equation, Compositio Math. 91 (1994), 201-221. 